\title{
Hydrogeochemistry of Hot Springs and the 2018 Mojiang M 5.9 Earthquake-Related Chemical Changes in the Simao Basin, China
}

\section{Qilin Li ( $\sim$ qilinli1988@163.com )}

Yunnan Earthquake Administration https://orcid.org/0000-0002-3603-5788

\section{Ciping Zhao}

Yunnan Earthquake Administration

\section{Yun Wang}

Yunnan Earthquake Administration

\section{Yiying Zhou}

Yunnan Earthquake Administration

\section{Hua Ran}

Yunnan Earthquake Administration

\section{Research article}

Keywords: hot spring, earthquake, hydrochemical characteristics, reservoir temperature, chemical changes

Posted Date: June 4th, 2021

DOl: https://doi.org/10.21203/rs.3.rs-569688/v1

License: (9) (1) This work is licensed under a Creative Commons Attribution 4.0 International License. Read Full License

Version of Record: A version of this preprint was published at Frontiers in Earth Science on January 12th, 2022. See the published version at https://doi.org/10.3389/feart.2021.717680. 


\title{
Hydrogeochemistry of hot springs and the 2018 Mojiang M 5.9 earthquake-
}

\section{related chemical changes in the Simao Basin, China}

\author{
Qilin Li *, Ciping Zhao, Yun Wang, Yiying Zhou, and Hua Ran
}

\section{Abstract}

Due to strong tectonic activity, the Simao Basin frequently experiences earthquakes with a magnitude of 6 or greater. This study investigated the hydrochemical characteristics of 21 thermal springs in the Simao Basin from 2018 to 2020 to determine how the 2018 Mojiang M5.9 earthquake caused hydrochemical changes. The results showed that the Simao Basin contained saline spring waters, $\mathrm{HCO}_{3}{ }^{-}$-rich spring waters, and $\mathrm{SO}_{4}{ }^{2-}$-rich spring waters. Stratum lithology characteristics in the area exerted obvious control over the water chemistry characteristics. Saline springs flowed through red beds and dissolved large amounts of halite, which is rich with $\mathrm{Cl}^{-}$and $\mathrm{Na}^{+} . \mathrm{Ca}^{2+}\left(\mathrm{Mg}^{2+}\right)$ and $\mathrm{HCO}_{3}^{-}$in the hot spring waters were mainly derived from the dissolution of carbonate minerals, gypsum, and anhydrite in Triassic rocks. The higher $\mathrm{SO}_{4}{ }^{2-}$ content in the hot spring waters was influenced by pyrite contained in Ailaoshan metamorphic rocks. The reservoir temperatures $\left(121-289^{\circ} \mathrm{C}\right)$ in the Simao Basin were estimated by the silica-enthalpy mixing model equation and silicaenthalpy diagram. The hot springs with higher reservoir temperatures $\left(>250^{\circ} \mathrm{C}\right)$ were mainly located at the edges of the basin, which may correlate with nearby deep faults that provided deep heat and metamorphic rocks with low permeabilities. The concentrations of $\mathrm{Ca}^{2+}$ and $\mathrm{HCO}_{3}{ }^{-}$in most springs increased obviously before the Mojiang M5.9 earthquake but decreased after the earthquake. Changes also occurred in the reservoir temperatures of some spring samples. These hydrogeochemical variations might be attributable to accelerated water-rock interactions and/or the mixing of second fluids due to the earthquake.

Keywords: hot spring, earthquake, hydrochemical characteristics, reservoir temperature, chemical changes

\section{Introduction}

The Simao Basin (the southern half of the Lanping-Simao Basin) located in the Yunnan-Tibet geothermal belt is a typical red-bed Basin with strong tectonic activity (Figs. 1a and b). Various types and quantities of springs exist in the basin. The hydrochemical characteristics of hot springs help reveal information about geothermal reservoirs and thermal groundwater circulation and may provide insight into potential geothermal resources and the subsurface transport of substances. Some studies on springs in the Simao Basin have focused on potassium exploration through salty and saline *Correspondence: e-mail:qilinli1988@163.com 
springs, the exploration and utilization of geothermal resources, and the origin and evolution of the springs were also examined [1-4]. However few reports have studied the earthquake-related composition of groundwater characteristics and changes in the basin. The study area is located in the Simao-Pu'er seismic zone, which is one of the most important strong earthquake disaster areas in the Yunnan province [5]. It is helpful to study the hydrochemical characteristics and changes in the hot springs in order to understand the breeding process of strong earthquakes in this district.

Earthquake-related groundwater chemical composition changes have been widely used in the search for seismic precursors. Tsunogai et al. [6] observed increased $\mathrm{Cl}^{-}$and $\mathrm{SO}_{4}{ }^{2-}$ concentrations in groundwater prior to the Kobe earthquake in Japan. Claesson et al. [7] observed a rapid increase in multicomponent constituents and a decrease in the $\mathrm{Na} / \mathrm{Ca}$ content of groundwater due to a seismic event $(\mathrm{M}=5.8)$ in northern Iceland. Changes in the concentrations of $\mathrm{Ca}^{2+}$ and $\mathrm{HCO}_{3}{ }^{-}$ were observed before and after the Wenchuan earthquake $(M s=8.0)$, the Lushan earthquake $(M s=7.0)$, and the Kangding earthquake $(\mathrm{Ms}=6.3)$ in China $[8,9]$. Seismic hydrochemical changes may be linked to changes in the permeability structure, the mixture of deep and shallow fluids, and/or fluid-rock interactions caused by tectonic activity [10,11].

Here, new hydrochemical data from hot springs in the Simao Basin are reported. We investigated the hydrochemical characteristics of these thermal springs from 2018 to 2020. During the study period, the 2018 Mojiang M5.9 earthquake occurred in the study area and caused significant hydrochemical changes. Earthquake-related hydrochemical changes were studied and the possible mechanisms in the geothermal area were explained.

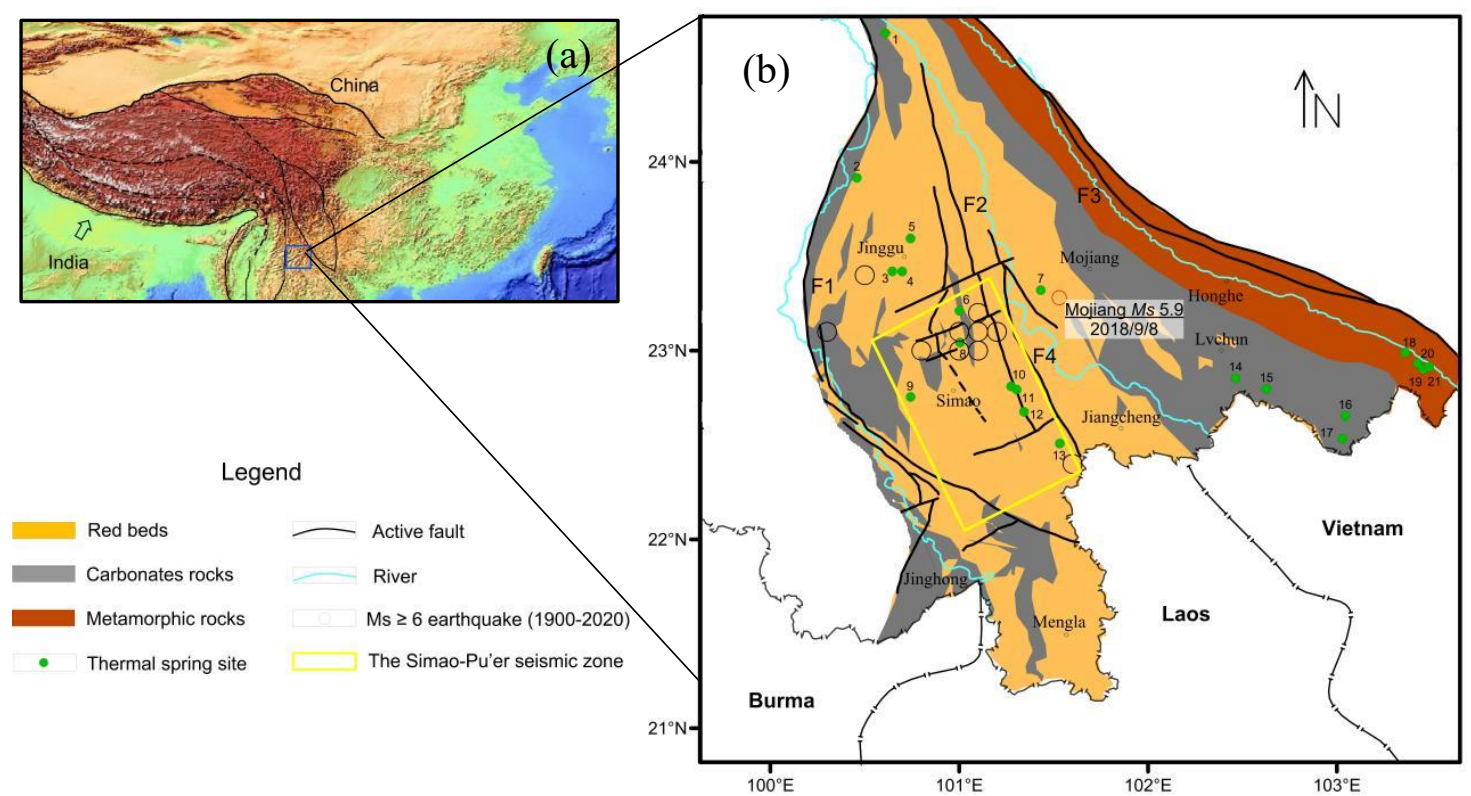

47 Fig. 1. Topographic map of the study area (a) and schematic tectonics and spring sites in the Simao Basin (b; modified from Zhang et al.) [12]. Abbreviations: F1 = Lancangjiang fault, F2 = Wuliangshan fault, F3 = Ailaoshan fault, F4 = Mohei fault. 


\section{Geological setting}

The Simao basin is located in southwest Yunnan, China and belongs to the southern section of the Sanjiang fold system, which is narrow in the north and wide in the south in the shape of a broom (Figs. 1a and b) [13]. Several huge NW-SE and nearly S-N trending faults control the formation and tectonic evolution of the basin. The Lancangjiang deep fault $(\mathrm{F} 1)$ in a roughly S-N direction and the Ailaoshan deep fault (F3) in a NW-SE direction are the western and eastern margins of the basin, respectively. A series of NW-SE trending faults have developed in the basin-for instance, the Wuliangshan fault (F2) and the Mohei fault (F4) (Fig. 1) [14]. Most parts of the Simao Basin are underlain by thick Mesozoic and Cenozoic red beds. Proterozoic metamorphic rocks are only exposed in the margin of the basin. The Upper and Middle Paleozoic limestones outcrop in the central block uplifts and the marginal belt uplifts in the basin [15]. According to seismic records, many moderate to strong crustal earthquakes have occurred in the basin, including the 2007 Ning'er earthquake (Ms 6.4), the 2014 Jinggu earthquake (Ms 6.6, Ms 5.8, and Ms 5.9), and the 2018 Mojiang earthquake (Ms 5.9) (http://www.ceic.ac.cn/). The Simao Basin is situated in the southern Yunnan-Tibet geothermal zone, which shows strong geothermal activity. The active crustal movement creates favorable conditions for the formation of hightemperature geothermal fields [16] with a terrestrial heat flow of $63.2 \mathrm{MW} / \mathrm{m}^{2}$ [17]. The distribution of the banded geothermal wells are controlled by the tectonic activities that formed deeper fracture zones, which can provide channels for the enrichment and migration of geothermal fluids.

\section{Materials and methods}

Hot springs were geochemically investigated in the Simao basin from 2018 to 2020, and 38 hydrothermal water samples were obtained (sampling locations shown in Fig. 1b). Based on previous studies, we collected more hydrochemical data from these thermal spring samples in the 1980s and in 2017. The sampling locations and information are listed in Table 1. The field investigations include on-site measurements of $\mathrm{pH}$, temperature, electrical conductivity (EC), which were measured with a portable multi-parameter water quality analyzer (produced by WTW, Germany). The precision of these factors were $0.01,0.1^{\circ} \mathrm{C}$, and $1 \mu \mathrm{S} \cdot \mathrm{cm}^{-1}$, respectively. During sampling, the water samples for analysis were filtered through a $0.45-\mu \mathrm{m}$ membrane filter and then stored in plastic bottles. All samples were measured at the deep-earth fluids laboratory, Yunnan Earthquake Administration, China. Cations $\left(\mathrm{Na}^{+}, \mathrm{K}^{+}, \mathrm{Ca}^{2+}, \mathrm{Mg}^{2+}, \mathrm{Li}^{+}, \mathrm{NH}_{4}^{+}, \mathrm{Rb}^{+}, \mathrm{Cs}^{+}, \mathrm{Sr}^{2+}\right)$ and anions $\left(\mathrm{F}^{-}, \mathrm{Cl}^{-}, \mathrm{NO}_{2}^{-}, \mathrm{Br}^{-}, \mathrm{NO}_{3}^{-}, \mathrm{SO}_{4}{ }^{2-}\right)$ were tested by ion chromatography (883 Basic IC plus, Metrohm, Switzerland; detection limit: $\left.0.01 \mathrm{mg} \cdot \mathrm{L}^{-1}\right) . \mathrm{SiO}_{2}(\mathrm{aq}), \mathrm{HCO}_{3}{ }^{-}$, and $\mathrm{CO}_{3}{ }^{2-}$ were detected according to the China National Standard Examination Methods for Drinking Natural Mineral Water (GB/T 8538-2008). $\mathrm{SiO}_{2}$ (aq) was measured by a visible spectrophotometer, and $\mathrm{HCO}_{3}{ }^{-}$and $\mathrm{CO}_{3}{ }^{2-}$ by the volumetric method ( $\mathrm{HCl}$ titration). 

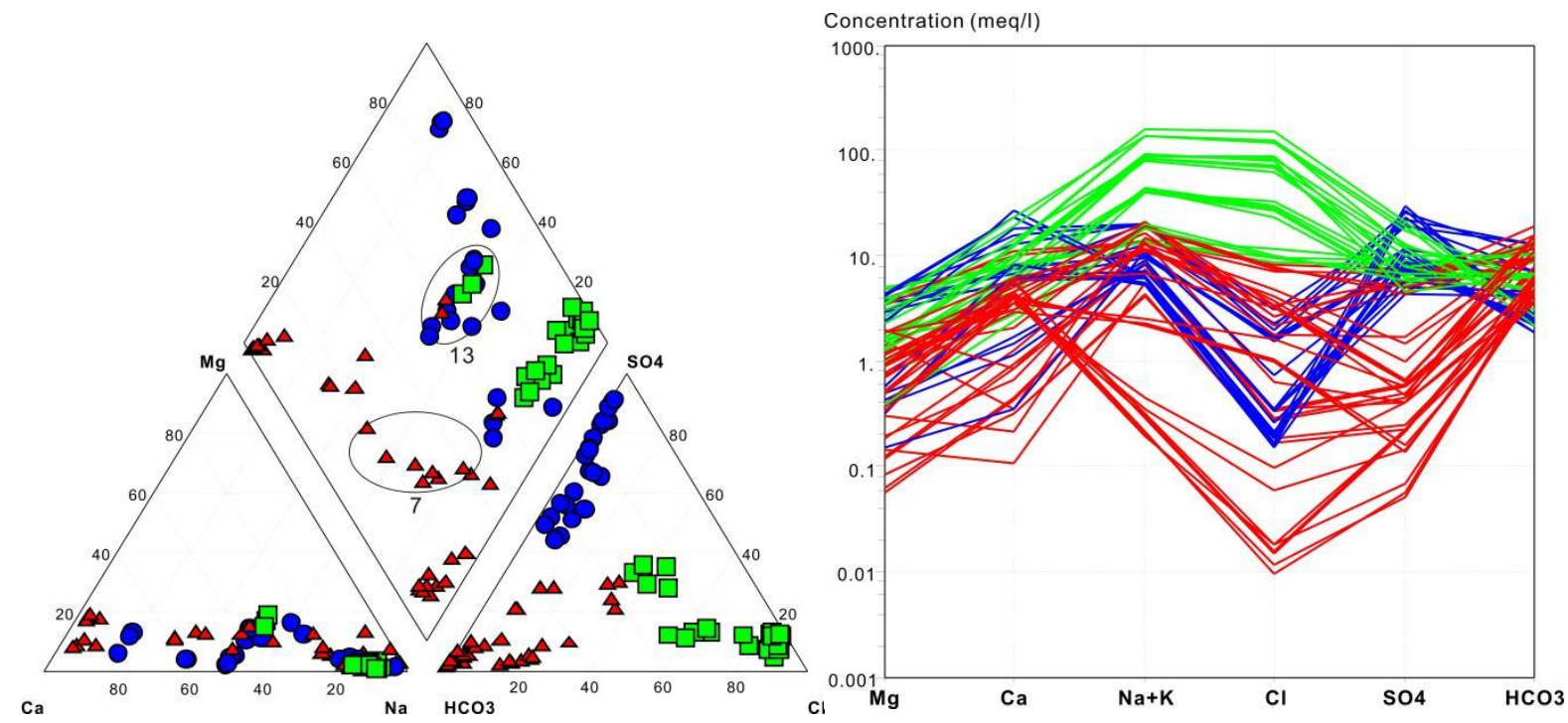

Fig. 2. Piper diagram (a) and Schoeller diagram (b) of the geothermal waters. Process (black circles) indicating the changes before and after the Mojiang earthquake.

The physicochemical parameters and geochemical data of hot springs in the Simao basin are listed in Table 1 and

Table 2. The 21 hot springs had temperatures ranging between $30^{\circ} \mathrm{C}$ and $102{ }^{\circ} \mathrm{C}$. The $\mathrm{pH}$ values and total dissolved solids (TDS) were 5.60-8.26 and 237-10444 mg/L, respectively. The saline spring samples (No. 3, 4, and 5) had relatively higher TDS values (5351-10444 mg/L) than others. Based on the data for ions $\left(\mathrm{K}^{+}, \mathrm{Na}^{+}, \mathrm{Ca}^{2+}, \mathrm{Mg}^{2+}, \mathrm{Cl}^{-}, \mathrm{SO}_{4}^{2-}\right.$, and $\left.\mathrm{HCO}_{3}^{-}\right)$in the thermal springs from the 1980s to 2020, the Piper and Schoeller diagrams (Figs. 2) show the concentrations of major and minor ions in springs. In the Piper diagram, the 21 water samples are roughly divided into three groups. Saline spring waters are of $\mathrm{Na}-\mathrm{Cl}$ type. Samples containing $\mathrm{HCO}_{3}{ }^{-}$as the predominant anion $\left(\mathrm{HCO}_{3}{ }^{-}>\mathrm{SO}_{4}{ }^{2-}>\mathrm{Cl}^{-}\right.$or $\mathrm{HCO}_{3}{ }^{-}>\mathrm{Cl}^{-}>$ $\mathrm{SO}_{4}{ }^{2-}$ ) include $\mathrm{Na}-\mathrm{HCO}_{3}, \mathrm{Ca}-\mathrm{HCO}_{3}, \mathrm{Ca}-\mathrm{Na}-\mathrm{HCO}_{3}, \mathrm{Na}-\mathrm{Ca}-\mathrm{HCO}_{3}, \mathrm{Na}-\mathrm{HCO}_{3}-\mathrm{SO}_{4}, \mathrm{Na}-\mathrm{HCO}_{3}-\mathrm{Cl}, \mathrm{Na}-\mathrm{Ca}-\mathrm{HCO}_{3}-\mathrm{SO}_{4}$, and $\mathrm{Na}-\mathrm{HCO}_{3}-\mathrm{Cl}-\mathrm{SO}_{4}$. Samples containing $\mathrm{SO}_{4}{ }^{2-}$ as the predominant anion $\left(\mathrm{SO}_{4}{ }^{2-}>\mathrm{HCO}_{3}{ }^{-}>\mathrm{Cl}^{-}\right.$or $\left.\mathrm{SO}_{4}{ }^{2-}>\mathrm{Cl}^{-}>\mathrm{HCO}_{3}{ }^{-}\right)$ include Ca-SO ${ }_{4}, \mathrm{Na}-\mathrm{Ca}-\mathrm{SO}_{4}, \mathrm{Ca}-\mathrm{Na}-\mathrm{SO}_{4}, \mathrm{Na}-\mathrm{SO}_{4}-\mathrm{HCO}_{3}$, and Na-Ca- $\mathrm{SO}_{4}-\mathrm{HCO}_{3}$. Other ions $\left(\mathrm{Li}^{+}, \mathrm{NH}_{4}^{+}, \mathrm{Rb}^{+}, \mathrm{Cs}^{+}, \mathrm{Sr}^{2+}, \mathrm{F}^{-}\right.$, $\mathrm{NO}_{2}^{-}, \mathrm{Br}^{-}, \mathrm{NO}_{3}^{-}$) were present in relatively low concentrations. The components $\mathrm{F}^{-}$, and $\mathrm{Sr}^{2+}$ of data points 14-21 had relatively higher concentrations, which may suggest that these spring waters undergo deep circulation.

Fig. 3 shows the relationships between major ions of the spring water, i.e. $\mathrm{HCO}_{3}{ }^{-}$vs. $\mathrm{Na}^{+}, \mathrm{Na}^{+}$vs. $\mathrm{Cl}^{-}, \mathrm{SO}_{4}{ }^{2-}$ vs. $\mathrm{Ca}^{2+}$, $\mathrm{HCO}_{3}{ }^{2-}$ vs. $\mathrm{Ca}^{2+},\left(\mathrm{Ca}^{2+}+\mathrm{Mg}^{2+}\right)$ vs. $\left(\mathrm{HCO}_{3}{ }^{-}+\mathrm{SO}_{4}{ }^{2-}\right)$ and $\mathrm{SO}_{4}{ }^{2-}$ vs. $\mathrm{Cl}^{-}$. Except for data points $3,4,5,8$, 9, and 12, the majority of data points plot close to the 1:1 line in Figure 3(a), indicating that the quality of $\mathrm{Na}$ is influenced by the 
dissolution of Na-feldspar. Samples 8 and 9 fall to the lower-right of the 1:1 line, which means that $\mathrm{HCO}_{3}{ }^{-}$has another source. Fig. 3d illustrates that carbonate minerals (calcite and dolomite) are also a source of $\mathrm{HCO}_{3}{ }^{-}$. The values of $\mathrm{HCO}_{3}{ }^{-}$ and $\mathrm{Na}^{+}$in saline spring waters of 3, 4, 5, and 12 are much greater than 1, suggesting that the dissolution of Na-feldspar is not the main source of these ions (Fig. 3a). Spring samples 3, 4, and 5 are located in Jinggu County, which has large halite deposits [13]. In Figure 3(b), saline spring samples of 3, 4, 5, and 12 with high $\mathrm{Cl}^{-}$and $\mathrm{Na}^{+}$contents lie on the dissolution line of $\mathrm{NaCl}$, showing that halite is the main source of Na. Except for data points 6, 7, 8, and 9 in the Triassic carbonate rocks, most water falls near the 1:1 line (gypsum dissolution line), suggesting that the dissolution of gypsum and anhydrite is the main source of $\mathrm{Ca}^{2+}$ and $\mathrm{SO}_{4}{ }^{2-}$ (Fig. 3c). Data points of 6, 7, 8, and 9 fall above the trend lines of $\mathrm{SO}_{4}{ }^{2-}$ and $\mathrm{Ca}^{2+}$, demonstrating that there is another source of $\mathrm{Ca}^{2+}$ apart from gypsum and anhydrite. The Triassic carbonate strata in the Simao Basin are located under the Jurassic red bed. The spring water flows through carbonate formations and can dissolve calcite and dolomite. Anhydrite is distributed in the thin interlayer of the salt series, and gypsum is distributed in the mud conglomerate layer on the surface [14]. The ionic ratios of $\left(\mathrm{Ca}^{2+}+\mathrm{Mg}^{2+}\right)$ vs. $\left(\mathrm{HCO}_{3}{ }^{-}+\mathrm{SO}_{4}{ }^{2-}\right)$ in Figure 3e show that the dissolution of dolomite, calcite, gypsum, and anhydrite is responsible for the production of $\mathrm{Ca}^{2+}, \mathrm{Mg}^{2+}, \mathrm{SO}_{4}{ }^{2-}$, and $\mathrm{HCO}_{3}{ }^{-}$. A correlation between $\mathrm{Cl}^{-}$and $\mathrm{SO}_{4}{ }^{2-}$ can be inferred from Fig. 3f. The positive chloride-sulfate relation probably reflects the mixing of geothermal water and cold water. Atmospheric oxygen in the cold water oxidizes $\mathrm{H}_{2} \mathrm{~S}$ to $\mathrm{SO}_{4}{ }^{2-}$ at shallow depths, which increases the $\mathrm{SO}_{4}{ }^{2-}$ concentration of the geothermal water, (e.g., sampling points 10,11, 18, 19, 20, and 21). In addition, the lithology suggests that the dissolution of the pyrite-bearing formation rocks near the Ailaoshan fault may increase the $\mathrm{SO}_{4}{ }^{2-}$ content in the groundwater [18].
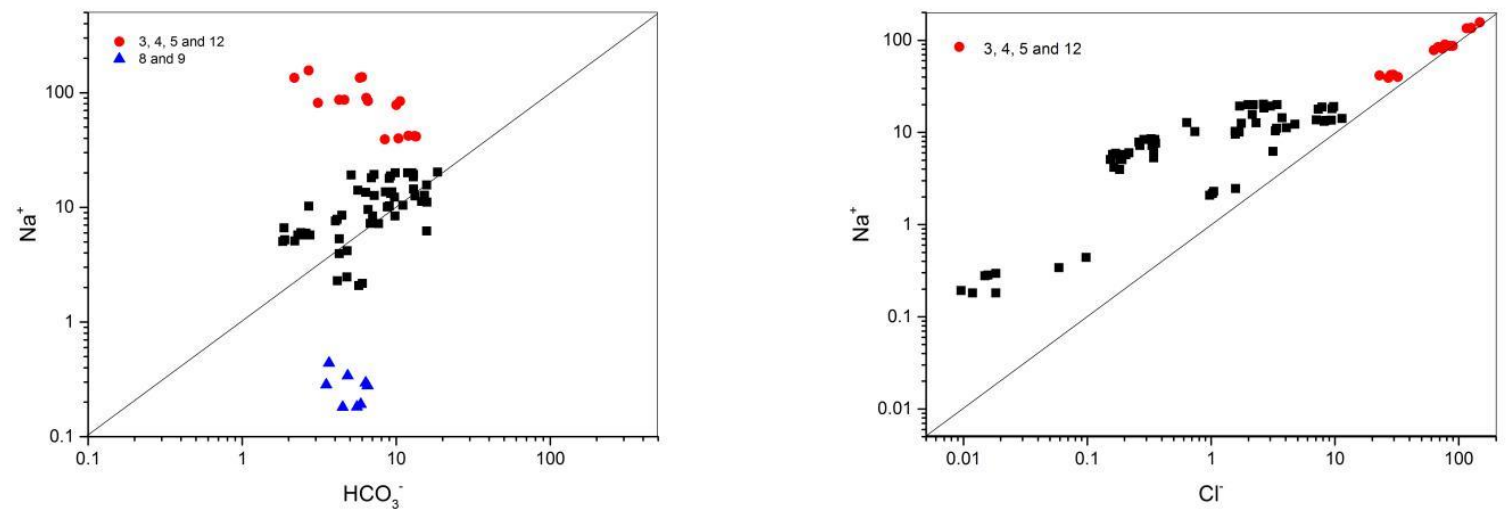

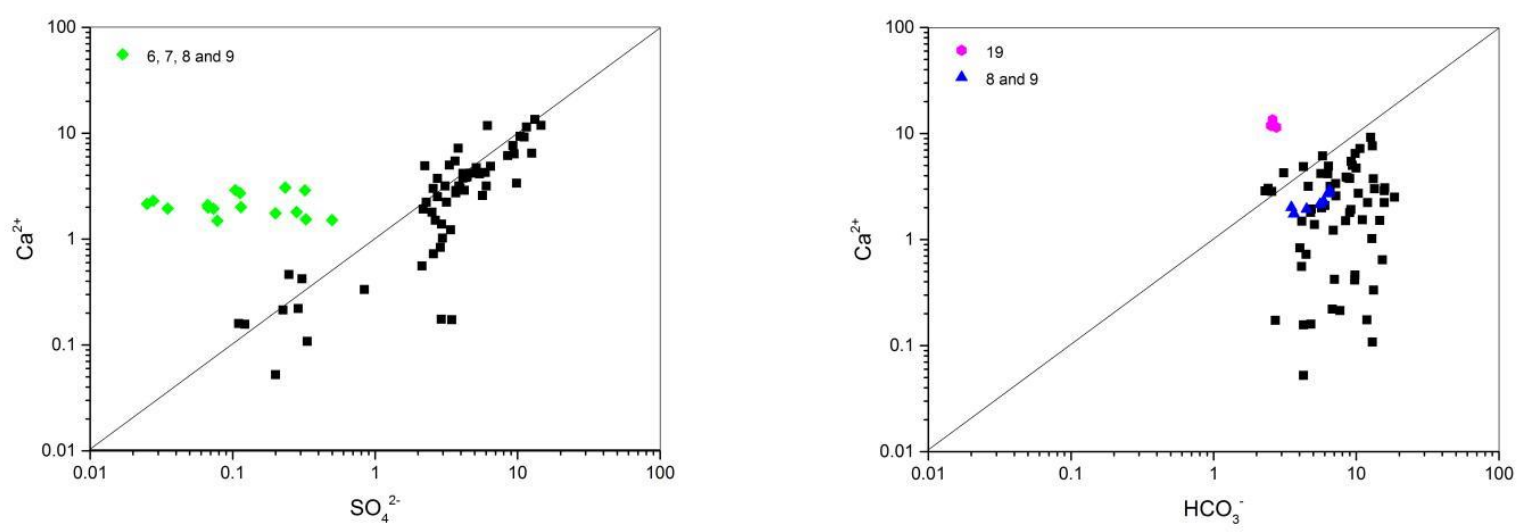

120
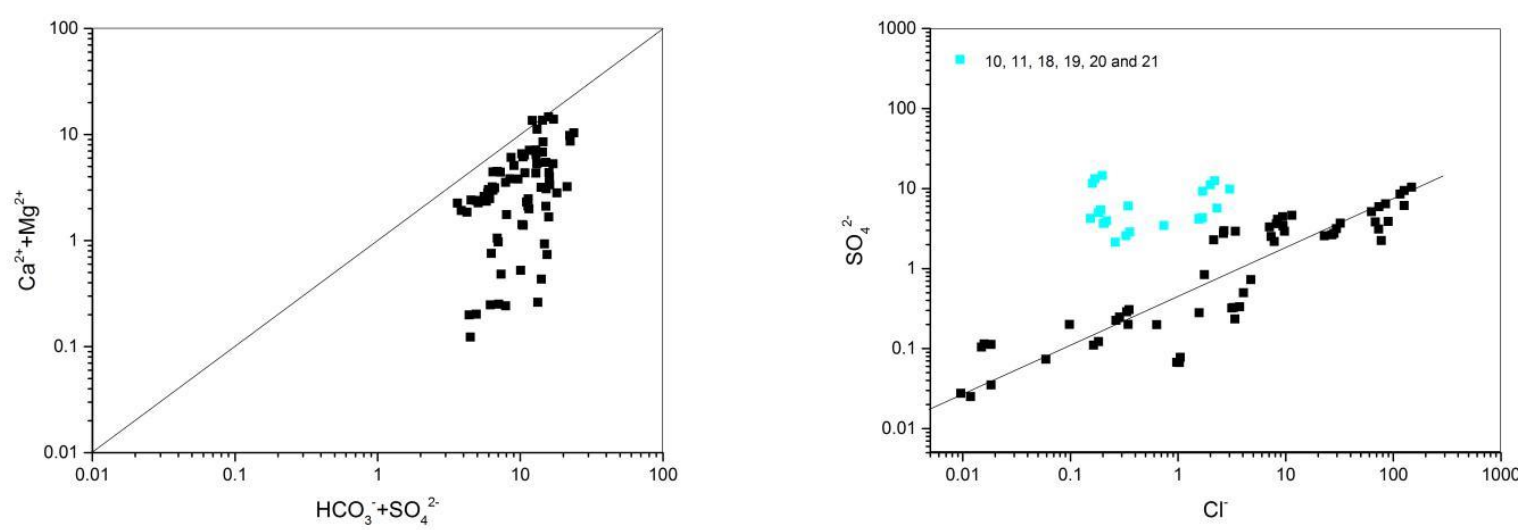

Fig. 3. Relationship plots of major ions of the spring water in the Simao Basin.

The waters in different springs have the same chemical characteristics, which indicates they may be derived from the same source. As shown in the Piper diagram (Fig. 2), the water chemistry in No.7 and 13 changed during the study period. No.7 is located in the near-field of the Mojiang earthquake. The water chemistry in No.7 changed from $\mathrm{Na}-\mathrm{HCO}-\mathrm{Cl}$ to $\mathrm{Na}-\mathrm{Ca}-\mathrm{HCO}_{3}$ in June 2018 before the Mojiang M5.9 earthquake on September 8 and from Na-Ca- $\mathrm{HCO}_{3}$ back to $\mathrm{Na}_{-}-\mathrm{HCO}_{3}-$ $\mathrm{Cl}$ in 2020. Similarly, the water chemistry in No.13 changed from Na-Ca-Cl-SO $4-\mathrm{HCO}_{3}$ to $\mathrm{Na}-\mathrm{Ca}-\mathrm{HCO}-\mathrm{Cl}-\mathrm{SO}_{4}$ before the Mojiang M5.9 earthquake and from Na-Ca-HCO $3-\mathrm{Cl}-\mathrm{SO}_{4}$ back to $\mathrm{Na}-\mathrm{Ca}-\mathrm{Cl}-\mathrm{SO}_{4}-\mathrm{HCO}_{3}$ after the earthquake; however, no significant changes were found in other spring samples (Table 2). The time series of anion and cation ions for these springs were plotted. $\mathrm{Ca}^{2+}$ and $\mathrm{HCO}_{3}{ }^{-}$ions in the springs exhibited significant changes before the Mojiang M5.9 earthquake, while other ions stayed relatively stable. We found that the concentrations of $\mathrm{Ca}^{2+}$ and $\mathrm{HCO}_{3}{ }^{-}$in most springs (blue lines) increased obviously before the Mojiang M5.9 earthquake but decreased after the earthquake (Fig. 4). Data points 1, 2, 6, $8,9,18,19,20$, and 21 (black lines) changed slightly before and after the earthquake. The $\mathrm{Na} / \mathrm{Ca}$ ratio appears to be 
136 sensitive to the changing stresses associated with $\mathrm{M}>4$ earthquakes [7]. In Fig. 4, the $\mathrm{Na} / \mathrm{Ca}$ data decreased before the

137 Mojiang M5.9 earthquake. After the earthquake, the $\mathrm{Na} / \mathrm{Ca}$ ratio increased slightly and then recovered in 2020.

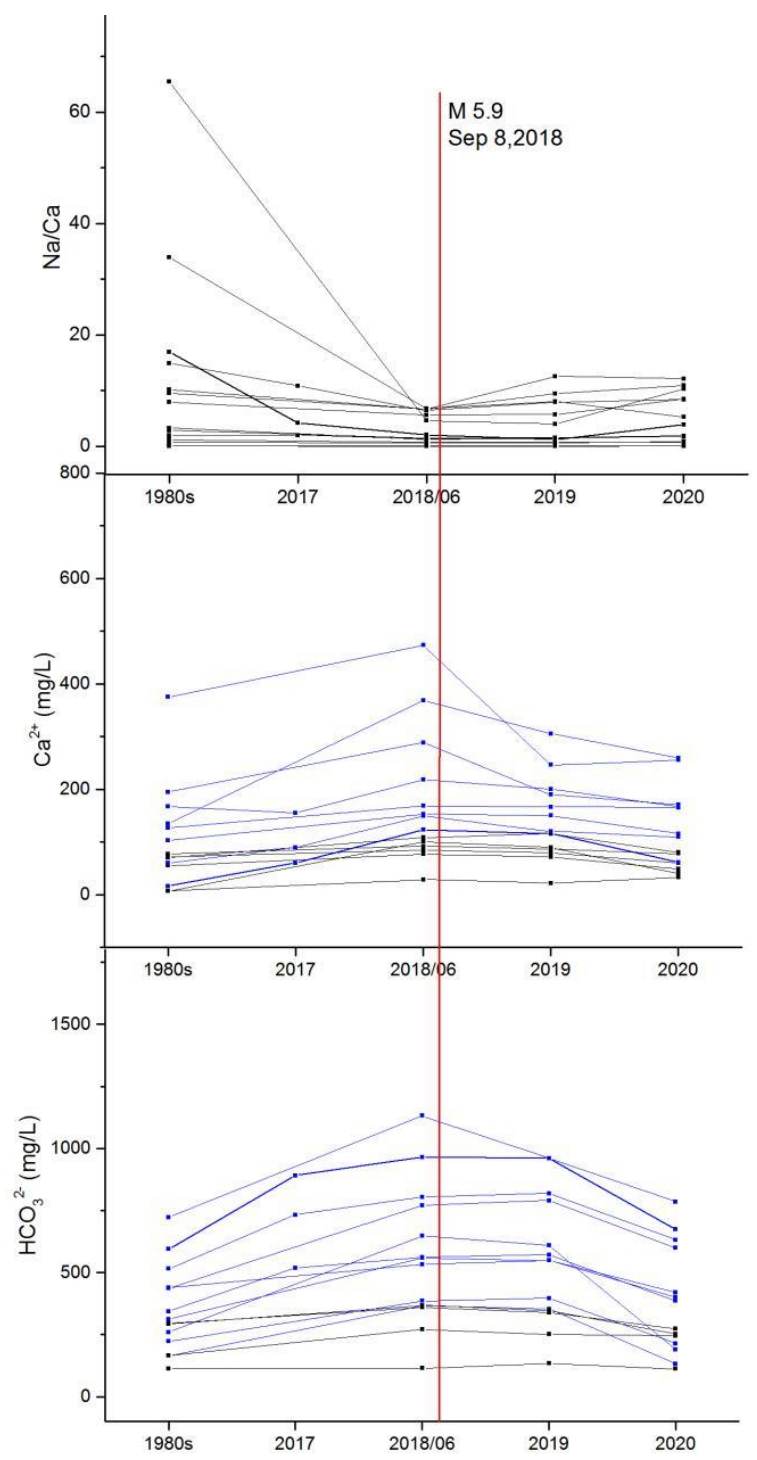

138

Fig. 4. Hydrogeochemical changes of the thermal springs from the 1980 s to 2020.

141 Table 1

142 Location, physicochemical parameters, and water type of the thermal springs in the Simao Basin.

\begin{tabular}{|c|c|c|c|c|c|c|c|c|c|}
\hline Sample No. & Site Name & Long. $\left(\mathrm{E}^{\circ}\right)$ & Lat. $\left(\mathrm{N}^{\circ}\right)$ & Time & $T$ & pH & EC & TDS & Water Type $^{a}$ \\
\hline $1 \mathrm{a}$ & \multirow{4}{*}{ Zhongcang hot spring } & \multirow{4}{*}{100.609226} & \multirow{4}{*}{24.681837} & $1982 / 12$ & 43.5 & 7.00 & - & 1344 & $\mathrm{Na}-\mathrm{HCO} 3-\mathrm{SO} 4$ \\
\hline $1 \mathrm{~b}$ & & & & $2018 / 6$ & 47.5 & 6.37 & 2400 & 2090 & $\mathrm{Na}-\mathrm{HCO} 3-\mathrm{SO} 4$ \\
\hline $1 \mathrm{c}$ & & & & $2019 / 3$ & 31.2 & 6.68 & 2030 & 1741 & $\mathrm{Na}-\mathrm{Ca}-\mathrm{HCO} 3-\mathrm{SO} 4$ \\
\hline $1 d$ & & & & $2020 / 6$ & 30.1 & 6.69 & 2300 & 1664 & $\mathrm{Na}-\mathrm{HCO} 3-\mathrm{SO} 4$ \\
\hline $2 \mathrm{a}$ & \multirow{2}{*}{ Xiejiatian hot spring } & \multirow{2}{*}{100.458191} & \multirow{2}{*}{23.915453} & $1982 / 12$ & 51.5 & 7.00 & - & 1399 & $\mathrm{Na}-\mathrm{Cl}-\mathrm{SO} 4$ \\
\hline $2 b$ & & & & $2018 / 6$ & 49.8 & 6.74 & 2290 & 1574 & $\mathrm{Na}-\mathrm{HCO} 3-\mathrm{Cl}$ \\
\hline
\end{tabular}




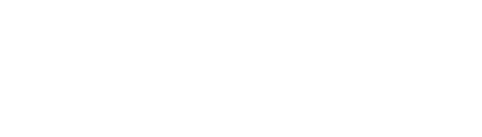

Mangfei spring

$100.645755 \quad 23.420093$

(n)

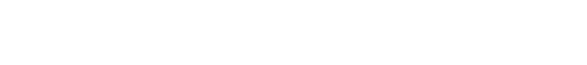

$\begin{array}{llll}2019 / 3 & 49.8 & 6.83 & 2280 \\ 2020 / 6 & 50.8 & 6.89 & 2560 \\ 2018 / 6 & 38.0 & 6.75 & 10000 \\ 2019 / 3 & 36.6 & 6.90 & 10470\end{array}$

$\begin{array}{lllll}2020 / 6 & 34.7 & 6.75 & 11230 & 6001\end{array}$

Na-HCO3-Cl-SO4

$\mathrm{Na}-\mathrm{Cl}-\mathrm{HCO} 3-\mathrm{SO} 4$

$\mathrm{Na}-\mathrm{Cl}$

$\mathrm{Na}-\mathrm{Cl}$

$\mathrm{Na}-\mathrm{Cl}$

$\mathrm{Na}-\mathrm{Cl}$

$\mathrm{Na}-\mathrm{Cl}$

$\mathrm{Na}-\mathrm{Cl}$

$\mathrm{Na}-\mathrm{Cl}$

$\mathrm{Na}-\mathrm{Cl}$

$\mathrm{Na}-\mathrm{Cl}$

$\mathrm{Na}-\mathrm{Cl}$

$\mathrm{Na}-\mathrm{Cl}$

Ca-Na-HCO3

Ca-Na-HCO3

$\mathrm{Ca}-\mathrm{Na}-\mathrm{HCO} 3$

Ca-Na-HCO3

$\mathrm{Na}-\mathrm{HCO} 3-\mathrm{Cl}$

$\mathrm{Na}-\mathrm{HCO} 3-\mathrm{Cl}$

$\mathrm{Na}-\mathrm{Ca}-\mathrm{HCO} 3$

$\mathrm{Na}-\mathrm{Ca}-\mathrm{HCO} 3$

$\mathrm{Na}-\mathrm{HCO} 3-\mathrm{Cl}$

$\mathrm{Ca}-\mathrm{HCO} 3$

$\mathrm{Ca}-\mathrm{HCO} 3$

$\mathrm{Ca}-\mathrm{HCO} 3$

$\mathrm{Ca}-\mathrm{HCO} 3$

$\mathrm{Ca}-\mathrm{HCO} 3$

Ca-HCO3

$\mathrm{Ca}-\mathrm{HCO} 3$

$\mathrm{Ca}-\mathrm{HCO} 3$

Na-Ca-SO4-HCO3

$\mathrm{Na}-\mathrm{Ca}-\mathrm{SO} 4-\mathrm{HCO} 3$

$\mathrm{Na}-\mathrm{Ca}-\mathrm{SO} 4-\mathrm{HCO} 3$

$\mathrm{Na}-\mathrm{Ca}-\mathrm{SO} 4-\mathrm{HCO} 3$

$\mathrm{Na}-\mathrm{Ca}-\mathrm{SO} 4-\mathrm{HCO} 3$

$\mathrm{Na}-\mathrm{Ca}-\mathrm{HCO} 3-\mathrm{SO} 4$

$\mathrm{Na}-\mathrm{Ca}-\mathrm{HCO} 3-\mathrm{SO} 4$

$\mathrm{Na}-\mathrm{Ca}-\mathrm{SO} 4-\mathrm{HCO} 4$

$\mathrm{Na}-\mathrm{Cl}$

$\mathrm{Na}-\mathrm{Cl}-\mathrm{HCO} 3$

$\mathrm{Na}-\mathrm{Cl}-\mathrm{HCO} 3$

$\mathrm{Na}-\mathrm{Cl}-\mathrm{HCO} 3$

Na-Cl-HCO3

$\mathrm{Na}-\mathrm{Ca}-\mathrm{Cl}-\mathrm{SO} 4-\mathrm{HCO} 3$ 


\begin{tabular}{|c|c|c|c|c|c|c|c|c|c|}
\hline 13aa & & & & $2017 / 7$ & 40.8 & 5.60 & - & 1813 & $\mathrm{Na}-\mathrm{Ca}-\mathrm{Cl}-\mathrm{SO} 4-\mathrm{HCO} 3$ \\
\hline $13 b$ & & & & $2018 / 6$ & 35.0 & 6.84 & 2500 & 1770 & $\mathrm{Na}-\mathrm{Ca}-\mathrm{HCO} 3-\mathrm{Cl}-\mathrm{SO} 4$ \\
\hline $13 \mathrm{c}$ & & & & $2019 / 3$ & 35.0 & 6.94 & 2090 & 1715 & $\mathrm{Na}-\mathrm{Ca}-\mathrm{HCO} 3-\mathrm{Cl}-\mathrm{SO} 4$ \\
\hline $13 d$ & & & & $2020 / 6$ & 35.9 & 6.90 & 2850 & 1612 & $\mathrm{Na}-\mathrm{Ca}-\mathrm{Cl}-\mathrm{SO} 4-\mathrm{HCO} 3$ \\
\hline $14 \mathrm{a}$ & \multirow{3}{*}{ Pingkun spring } & \multirow{3}{*}{102.463591} & \multirow{3}{*}{22.8542} & $1983 / 12$ & 43.5 & 7.00 & - & 928 & $\mathrm{Na}-\mathrm{HCO} 3$ \\
\hline $14 \mathrm{c}$ & & & & $2019 / 3$ & 40.3 & 7.26 & 1397 & 1321 & $\mathrm{Na}-\mathrm{HCO} 3$ \\
\hline $14 \mathrm{~d}$ & & & & $2020 / 6$ & 42.0 & 7.33 & 1549 & 1273 & $\mathrm{Na}-\mathrm{HCO} 3$ \\
\hline $15 \mathrm{c}$ & \multirow{2}{*}{ Baha hot spring } & \multirow{2}{*}{102.626560} & \multirow{2}{*}{22.796782} & $2019 / 3$ & 72.0 & 8.00 & 810 & 694 & $\mathrm{Na}-\mathrm{HCO} 3$ \\
\hline $15 \mathrm{~d}$ & & & & $2020 / 6$ & 72.1 & 8.18 & 914 & 666 & $\mathrm{Na}-\mathrm{HCO} 3$ \\
\hline $16 \mathrm{a}$ & \multirow{3}{*}{ Puer hot spring } & \multirow{3}{*}{103.0441} & \multirow{3}{*}{22.653311} & $1983 / 12$ & 59.0 & 7.20 & - & 423 & $\mathrm{Na}-\mathrm{HCO} 3$ \\
\hline $16 \mathrm{c}$ & & & & $2019 / 3$ & 51.8 & 7.30 & 482 & 427 & $\mathrm{Na}-\mathrm{HCO} 3$ \\
\hline $16 \mathrm{~d}$ & & & & $2020 / 6$ & 51.8 & 7.25 & 553 & 391 & $\mathrm{Na}-\mathrm{HCO} 3$ \\
\hline $17 \mathrm{c}$ & \multirow{2}{*}{ Nanxing hot spring } & \multirow{2}{*}{103.028505} & \multirow{2}{*}{22.533045} & $2019 / 3$ & 65.0 & 7.99 & 962 & 870 & $\mathrm{Na}-\mathrm{HCO} 3$ \\
\hline $17 \mathrm{~d}$ & & & & $2020 / 6$ & 64.8 & 7.31 & 1123 & 754 & $\mathrm{Na}-\mathrm{HCO} 3$ \\
\hline $18 \mathrm{a}$ & \multirow{4}{*}{ Dadipeng hot spring } & \multirow{4}{*}{103.361475} & \multirow{4}{*}{22.990371} & $1983 / 12$ & 51.0 & 7.00 & - & 1074 & $\mathrm{Na}-\mathrm{Ca}-\mathrm{SO} 4$ \\
\hline $18 \mathrm{~b}$ & & & & $2018 / 6$ & 50.8 & 6.97 & 1270 & 914 & $\mathrm{Ca}-\mathrm{Na}-\mathrm{SO} 4$ \\
\hline $18 \mathrm{c}$ & & & & $2019 / 3$ & 48.1 & 7.44 & 1284 & 853 & $\mathrm{Ca}-\mathrm{Na}-\mathrm{SO} 4$ \\
\hline $18 \mathrm{~d}$ & & & & $2020 / 6$ & 50.8 & 7.00 & 1429 & 943 & $\mathrm{Ca}-\mathrm{Na}-\mathrm{SO} 4$ \\
\hline $19 \mathrm{~b}$ & \multirow{3}{*}{ Mapiyi spring } & \multirow{3}{*}{103.45726} & \multirow{3}{*}{22.902029} & $2018 / 6$ & 40.0 & 6.82 & 2450 & 2163 & $\mathrm{Ca}-\mathrm{SO} 4$ \\
\hline $19 \mathrm{c}$ & & & & $2019 / 3$ & 40.5 & 6.97 & 2480 & 1953 & $\mathrm{Ca}-\mathrm{SO} 4$ \\
\hline $19 d$ & & & & $2020 / 6$ & 40.6 & 6.99 & 2750 & 2246 & $\mathrm{Ca}-\mathrm{SO} 4$ \\
\hline $20 \mathrm{~b}$ & \multirow{3}{*}{ Malizhai hot spring } & \multirow{3}{*}{103.4285} & \multirow{3}{*}{22.931684} & $2018 / 6$ & 77.0 & 7.48 & 1162 & 823 & Na-Ca-SO4-HCO3 \\
\hline $20 \mathrm{c}$ & & & & $2019 / 3$ & 76.0 & 7.26 & 1280 & 788 & Na-Ca-SO4-HCO3 \\
\hline 20d & & & & $2020 / 6$ & 75.1 & 6.88 & 1375 & 782 & Na-Ca-SO4-HCO3 \\
\hline $21 \mathrm{a}$ & \multirow{4}{*}{ Mengping hot spring } & \multirow{4}{*}{103.488815} & \multirow{4}{*}{22.913696} & $1983 / 12$ & 102.2 & 7.50 & - & 915 & Na-SO4-HCO3 \\
\hline $21 \mathrm{~b}$ & & & & $2018 / 6$ & 94.0 & 8.26 & 1137 & 791 & Na-SO4-HCO3 \\
\hline $21 \mathrm{c}$ & & & & $2019 / 3$ & 93.0 & 7.90 & 1189 & 701 & Na-SO4-HCO3 \\
\hline $21 d$ & & & & $2020 / 6$ & 91.2 & 7.79 & 1270 & 799 & Na-SO4-HCO3 \\
\hline
\end{tabular}


144 Table 2

145 Chemical composition of the hot spring waters in the Simao Basin.

\begin{tabular}{|c|c|c|c|c|c|c|c|c|c|c|c|c|c|c|c|c|c|c|}
\hline & $\mathrm{Na}^{+}$ & $\mathrm{K}^{+}$ & $\mathrm{Ca}^{2+}$ & $\mathrm{Mg}^{2+}$ & $\mathrm{Li}^{+}$ & $\mathrm{NH}_{4}^{+}$ & $\mathrm{Rb}^{+}$ & $\mathrm{Cs}^{+}$ & $\mathrm{Sr}^{2+}$ & $\mathrm{F}^{-}$ & $\mathrm{Cl}^{-}$ & $\mathrm{NO}_{2}^{-}$ & $\mathrm{Br}^{-}$ & $\mathrm{NO}_{3}^{-}$ & $\mathrm{CO}_{3}{ }^{{ }^{-}}$ & $\mathrm{HCO}_{3}{ }^{-}$ & $\mathrm{SO}_{4}^{2^{2-}}$ & $\mathrm{SiO}_{2}$ \\
\hline $1 \mathrm{a}$ & 459.00 & 25.60 & 7.01 & 18.20 & 0.38 & 0.28 & 0.110 & 0.410 & & 1.67 & 121.00 & & & & 19 & 723 & 280.00 & 39 \\
\hline $1 \mathrm{c}$ & 361.01 & 13.16 & 89.40 & 14.05 & 0.42 & & & & 1.50 & 1.33 & 76.24 & 0.013 & 0.009 & 3.885 & & 961 & 219.49 & 29 \\
\hline $1 \mathrm{~d}$ & 423.87 & 11.78 & 40.93 & 15.53 & 0.47 & & & & 1.56 & 1.75 & 94.89 & 0.005 & 0.008 & 1.845 & & 786 & 285.32 & 39 \\
\hline $2 a$ & 440.00 & 18.10 & 55.50 & 8.90 & 0.84 & 0.88 & 0.050 & 0.150 & & 1.85 & 347.00 & & & & 13 & 311 & 281.00 & 67 \\
\hline $2 b$ & 432.58 & 7.20 & 76.72 & 4.76 & 0.99 & & 0.024 & 0.572 & 3.11 & 1.89 & 278.87 & & & 0.014 & & 559 & 208.64 & 69 \\
\hline $2 c$ & 411.39 & 9.09 & 71.94 & 4.54 & 0.93 & & 0.004 & 0.578 & 3.00 & 1.76 & 260.19 & 0.238 & 0.062 & 0.062 & & 549 & 241.04 & 60 \\
\hline $2 d$ & 415.08 & 6.99 & 48.98 & 4.61 & 0.93 & & & & 2.86 & 2.29 & 335.97 & & & 0.072 & & 421 & 325.64 & 67 \\
\hline $3 b$ & 2073.95 & 24.80 & 197.25 & 27.95 & 1.00 & & & & 0.00 & 1.31 & 2749.55 & & 0.062 & 1.054 & & 390 & 214.36 & 34 \\
\hline $3 c$ & 1949.40 & 20.12 & 127.26 & 15.05 & & & & & 3.65 & 1.51 & 2602.38 & & 0.464 & 0.939 & & 400 & 297.96 & 33 \\
\hline $3 d$ & 1995.10 & 20.79 & 127.01 & 15.59 & & & & & 3.72 & 1.62 & 3180.67 & & 0.556 & 3.425 & & 281 & 371.56 & 31 \\
\hline $4 a$ & 1995.00 & 34.10 & 195.00 & 37.30 & 0.45 & 1.02 & 0.140 & 0.360 & & 1.85 & 3013.00 & & & & & 260 & 620.00 & 45 \\
\hline $4 c$ & 1794.69 & 13.02 & 189.55 & 17.30 & & & & 0.718 & 4.05 & 1.14 & 2228.54 & & 0.233 & 0.025 & & 610 & 492.03 & 22 \\
\hline $4 d$ & 1874.56 & 13.82 & 170.73 & 20.14 & & & & & 4.38 & 0.58 & 2614.85 & 1.665 & 0.195 & 0.565 & & 189 & 569.35 & 43 \\
\hline $5 a$ & 3590.00 & 56.10 & 375.00 & 44.10 & 1.06 & 1.74 & 0.240 & 0.390 & & 2.32 & 5246.00 & & & & & 165 & 1000.00 & 39 \\
\hline $5 \mathrm{~b}$ & 3146.30 & 27.70 & 473.30 & 41.30 & 1.30 & & & & 7.00 & 0.68 & 4478.45 & & & 0.016 & & 366 & 590.11 & 37 \\
\hline $5 c$ & 3098.10 & 24.88 & 246.07 & 16.45 & & & & & 4.93 & 0.62 & 4105.40 & & 0.765 & 0.041 & & 354 & 820.10 & 35 \\
\hline $5 \mathrm{~d}$ & 3097.25 & 24.91 & 255.11 & 17.86 & & & & & 5.61 & 0.71 & 4474.50 & 0.675 & 0.012 & 1.016 & & 133 & 906.63 & 40 \\
\hline $6 a$ & 56.70 & 2.55 & 72.30 & 11.00 & 0.04 & 0.34 & & & & 0.50 & 55.90 & & & & & 292 & 27.00 & 33 \\
\hline $6 \mathrm{~b}$ & 49.85 & 1.78 & 84.05 & 9.29 & 0.02 & & & & 0.89 & 0.45 & 36.48 & & & 0.004 & & 368 & 6.42 & 32 \\
\hline $6 c$ & 47.93 & 1.71 & 79.62 & 8.56 & 0.03 & & & & 0.75 & 0.50 & 34.53 & & 0.002 & 0.006 & & 348 & 6.45 & 20 \\
\hline $6 \mathrm{~d}$ & 52.59 & 1.69 & 59.74 & 8.75 & 0.02 & & & & 1.11 & 0.66 & 37.35 & & 0.001 & 0.071 & & 253 & 7.49 & 28 \\
\hline $7 \mathrm{a}$ & 282.00 & 24.90 & 16.60 & 23.50 & 0.64 & 5.20 & 0.520 & 0.470 & & 1.12 & 169.00 & & & & & 595 & 70.00 & 56 \\
\hline $7 \mathrm{aa}$ & 259 & 19.6 & 60.7 & 14.2 & 0.47 & & 0.071 & & 1.75 & 0.32 & 144 & & 0.18 & 0.04 & & 891 & 47.90 & 47 \\
\hline $7 \mathrm{~b}$ & 255.71 & 19.13 & 123.04 & 22.27 & 0.78 & & 0.038 & 0.795 & 1.55 & 0.60 & 120.11 & & & & & 965 & 22.49 & 61 \\
\hline
\end{tabular}




\begin{tabular}{|c|c|c|c|c|c|c|c|c|c|c|c|c|c|c|c|c|c|}
\hline $7 \mathrm{~d}$ & 239.53 & 18.40 & 61.48 & 22.56 & 0.80 & & & & 1.33 & 0.58 & 117.18 & 0.001 & 0.085 & 0.157 & 673 & 31.38 & 52 \\
\hline $8 \mathrm{a}$ & 7.85 & 0.57 & 77.50 & 10.80 & 0.02 & 1.10 & & & & 0.09 & 2.10 & & & & 295 & 7.05 & 23 \\
\hline $8 \mathrm{~b}$ & 4.42 & 0.69 & 91.93 & 12.59 & & 0.04 & & & 0.27 & 0.06 & 0.34 & & & 0.480 & 359 & 2.66 & 19 \\
\hline $8 \mathrm{c}$ & 4.18 & 0.66 & 86.24 & 10.86 & & 0.04 & & & 0.19 & 0.08 & 0.42 & 0.003 & & 0.615 & 339 & 2.41 & 14 \\
\hline $8 \mathrm{~d}$ & 4.16 & 0.63 & 77.85 & 11.13 & & & & & 0.00 & 0.17 & 0.65 & & & 1.634 & 274 & 3.36 & 30 \\
\hline $9 \mathrm{a}$ & 10.10 & 0.15 & 70.20 & 4.10 & & 0.54 & & & & 0.42 & 3.48 & & & & 223 & 19.20 & 17 \\
\hline $9 b$ & 6.80 & 0.52 & 108.73 & 6.31 & 0.01 & 0.09 & & & 0.30 & 0.21 & 0.65 & & & 0.118 & 386 & 10.83 & 13 \\
\hline $9 \mathrm{c}$ & 6.42 & 0.51 & 116.00 & 5.88 & 0.01 & 0.05 & & & 0.17 & 0.17 & 0.53 & & 0.006 & 0.138 & 397 & 10.03 & 8 \\
\hline $9 \mathrm{~d}$ & 6.52 & 0.53 & 80.35 & 5.93 & 0.01 & 0.01 & & & 0.29 & 0.21 & 0.56 & & & 0.100 & 214 & 10.99 & 22 \\
\hline $10 \mathrm{a}$ & 445.00 & 23.70 & 135.00 & 46.20 & 0.13 & 1.70 & 0.480 & 0.540 & & 1.39 & 107.00 & & & & 437 & 945.00 & 50 \\
\hline $10 \mathrm{~b}$ & 459.90 & 15.64 & 368.21 & 28.82 & 0.23 & & 0.003 & 0.187 & 2.66 & 0.91 & 70.78 & & & 0.318 & 771 & 1067.17 & 48 \\
\hline $10 \mathrm{c}$ & 444.44 & 15.13 & 305.59 & 51.60 & 0.23 & & 0.022 & 0.222 & 2.72 & 0.69 & 60.27 & & 0.021 & 0.093 & 790 & 891.12 & 26 \\
\hline $10 \mathrm{~d}$ & 460.00 & 15.79 & 259.59 & 51.82 & 0.26 & & & & 2.57 & 0.85 & 77.65 & & & & 600 & 1205.83 & 37 \\
\hline $11 \mathrm{a}$ & 292.00 & 9.97 & 103.00 & 42.20 & 0.05 & 3.20 & 0.210 & 0.310 & & 0.59 & 82.00 & & & & 439 & 545.00 & 36 \\
\hline $11 \mathrm{~b}$ & 231.82 & 5.70 & 152.90 & 37.35 & 0.09 & & 0.008 & 0.145 & 1.24 & 0.37 & 59.83 & & 0.023 & 0.123 & 534 & 415.38 & 34 \\
\hline $11 \mathrm{c}$ & 236.27 & 5.71 & 150.24 & 37.22 & 0.10 & & & & 1.11 & 0.29 & 55.44 & & 0.001 & 0.526 & 549 & 397.88 & 19 \\
\hline $11 \mathrm{~d}$ & 220.49 & 5.36 & 115.77 & 34.90 & 0.11 & & & & 0.99 & 0.31 & 55.88 & & 0.013 & 0.003 & 400 & 406.79 & 16 \\
\hline $12 \mathrm{a}$ & 900.00 & 17.70 & 60.20 & 19.50 & 0.23 & 0.75 & 0.440 & 0.570 & & 0.92 & 957.00 & & & & 515 & 254.00 & 50 \\
\hline 12aa & 970 & 38.9 & 89.3 & 20.3 & 0.35 & & 0.076 & & 2.37 & & 1054 & & 0.35 & & 732 & 304.00 & 34 \\
\hline $12 b$ & 963.50 & 13.75 & 150.00 & 14.95 & 0.40 & 15.75 & & & 0.00 & 0.87 & 1003.20 & & & 0.039 & 804 & 263.31 & 60 \\
\hline $12 \mathrm{c}$ & 953.56 & 11.94 & 120.38 & 12.13 & & & & 0.694 & 1.96 & 0.66 & 814.04 & & 0.138 & 0.144 & 819 & 245.94 & 48 \\
\hline $12 \mathrm{~d}$ & 919.29 & 11.66 & 109.49 & 10.69 & & & & & 1.83 & 0.93 & 1145.34 & & 0.193 & 2.342 & 631 & 353.87 & 48 \\
\hline $13 \mathrm{a}$ & 325.00 & 7.22 & 168.00 & 57.50 & 0.03 & 0.74 & 0.190 & 0.430 & & 1.05 & 405.00 & & & & 344 & 445.00 & 39 \\
\hline 13aa & 313 & 6.81 & 155 & 61.7 & 0.05 & & 0.02 & & 1.48 & & 332 & & 0.07 & & 519 & 426.00 & 26 \\
\hline $13 \mathrm{~b}$ & 303.37 & 3.77 & 218.84 & 40.06 & 0.08 & & & & 1.08 & 0.34 & 291.15 & & & 0.087 & 562 & 349.21 & 34 \\
\hline $13 \mathrm{c}$ & 313.57 & 3.91 & 200.50 & 48.55 & 0.10 & & & & 1.23 & 0.29 & 251.59 & & & 0.423 & 572 & 318.67 & 28 \\
\hline $13 \mathrm{~d}$ & 310.93 & 3.72 & 167.23 & 47.26 & 0.10 & & & & 1.26 & 0.32 & 298.37 & & 0.041 & 0.026 & 386 & 397.06 & 32 \\
\hline $14 \mathrm{a}$ & 332.00 & 27.70 & 4.32 & 3.71 & 0.84 & 7.40 & 0.980 & 1.690 & & 10.20 & 133.00 & & & & 790 & 32.00 & 68 \\
\hline $14 \mathrm{c}$ & 293.82 & 18.53 & 25.84 & 2.23 & 0.83 & & 0.094 & 0.427 & 0.82 & 8.05 & 22.58 & & 0.040 & 0.037 & 929 & 19.07 & 61 \\
\hline $14 \mathrm{~d}$ & 289.32 & 15.88 & 13.36 & 2.36 & 0.85 & & 0.111 & 0.377 & 0.84 & 0.07 & 62.38 & & 0.012 & 0.041 & 807 & 80.70 & 51 \\
\hline $15 \mathrm{c}$ & 166.02 & 8.50 & 8.59 & 0.68 & 0.46 & & 0.111 & 0.378 & 0.14 & 9.78 & 9.44 & & 0.047 & & 468 & 21.64 & 101 \\
\hline
\end{tabular}




\begin{tabular}{|c|c|c|c|c|c|c|c|c|c|c|c|c|c|c|c|c|c|c|}
\hline $15 \mathrm{~d}$ & 166.51 & 8.53 & 8.83 & 0.74 & 0.46 & & 0.076 & 0.188 & 0.26 & 12.57 & 11.84 & 0.003 & 0.032 & 0.116 & 14 & 414 & 27.74 & 79 \\
\hline 16a & 122.00 & 13.20 & 2.10 & 1.70 & 0.10 & 4.05 & 0.740 & 0.570 & & 7.44 & 12.20 & & & & 13 & 260 & 19.20 & 95 \\
\hline $16 \mathrm{c}$ & 96.02 & 7.94 & 6.38 & 1.03 & 0.10 & & 0.101 & 0.156 & 0.23 & 6.30 & 5.83 & & & 0.008 & & 293 & 10.58 & 84 \\
\hline $16 \mathrm{~d}$ & 90.86 & 7.74 & 6.29 & 1.02 & 0.11 & & 0.095 & 0.113 & 0.24 & 7.08 & 6.49 & 0.003 & 0.012 & 0.059 & & 260 & 11.72 & 79 \\
\hline $17 \mathrm{c}$ & 193.19 & 13.12 & 18.49 & 1.50 & 0.40 & & 0.164 & 0.347 & 0.36 & 10.14 & 10.17 & 0.005 & 0.054 & 0.015 & & 598 & 23.80 & 94 \\
\hline $17 \mathrm{~d}$ & 193.39 & 13.27 & 16.90 & 1.45 & 0.41 & & 0.154 & 0.182 & 0.35 & 12.59 & 12.46 & 0.004 & 0.020 & 0.114 & 45 & 429 & 29.48 & 80 \\
\hline $18 \mathrm{a}$ & 152.00 & 13.10 & 127.00 & 8.60 & 0.05 & 3.84 & 0.300 & nd & & 3.70 & 12.20 & & & & & 114 & 580.00 & 116 \\
\hline $18 \mathrm{~b}$ & 119.87 & 8.55 & 168.30 & 6.91 & 0.10 & & 0.040 & 0.228 & 3.37 & 3.74 & 6.48 & & & 0.339 & & 115 & 480.87 & 101 \\
\hline $18 \mathrm{c}$ & 116.77 & 8.33 & 166.66 & 6.91 & 0.10 & & 0.032 & 0.250 & 3.28 & 3.33 & 5.45 & & & 0.028 & & 134 & 407.81 & 73 \\
\hline $18 \mathrm{~d}$ & 116.17 & 8.24 & 165.27 & 6.91 & 0.09 & & 0.103 & & 3.28 & 4.07 & 6.78 & & 0.004 & & & 112 & 519.55 & 84 \\
\hline $19 \mathrm{~b}$ & 136.97 & 14.75 & 540.65 & 27.75 & 0.38 & & 0.024 & 0.155 & 7.83 & 2.82 & 6.01 & & & 0.074 & & 158 & 1267.96 & 92 \\
\hline $19 \mathrm{c}$ & 132.22 & 12.90 & 459.22 & 52.47 & 0.34 & & & & 7.95 & 2.84 & 5.67 & & & & & 168 & 1111.44 & 47 \\
\hline $19 \mathrm{~d}$ & 132.47 & 12.74 & 475.14 & 49.59 & 0.38 & & & & 8.35 & 3.44 & 7.05 & & & & & 154 & 1402.24 & 87 \\
\hline $20 \mathrm{~b}$ & 138.34 & 14.72 & 121.52 & 3.96 & 0.15 & & 0.126 & 0.069 & 3.63 & 5.68 & 7.71 & & & 0.002 & & 147 & 379.40 & 151 \\
\hline $20 \mathrm{c}$ & 131.61 & 14.04 & 114.08 & 3.77 & 0.14 & & & & 3.14 & 4.79 & 7.18 & 0.013 & 0.006 & 0.001 & & 156 & 353.88 & 123 \\
\hline $20 \mathrm{~d}$ & 131.95 & 14.12 & 115.04 & 3.88 & 0.16 & & & & 3.23 & 5.20 & 7.29 & & 0.006 & & 7 & 140 & 353.46 & 139 \\
\hline $21 \mathrm{a}$ & 235.00 & 23.10 & 6.92 & 1.80 & 0.24 & 4.50 & 1.430 & 0.530 & & 8.13 & 26.30 & & & & & 165 & 332.00 & 191 \\
\hline $21 \mathrm{~b}$ & 195.87 & 20.52 & 29.06 & 5.88 & 0.27 & & 0.157 & & 0.61 & 9.36 & 11.65 & & 0.007 & 0.012 & & 271 & 246.37 & 156 \\
\hline $21 \mathrm{c}$ & 180.55 & 18.86 & 22.29 & 4.77 & 0.25 & & 0.159 & & 0.66 & 7.75 & 9.28 & & 0.022 & 0.023 & & 252 & 204.60 & 134 \\
\hline $21 \mathrm{~d}$ & 175.33 & 18.78 & 33.43 & 5.22 & 0.27 & & 0.135 & & 0.71 & 10.84 & 12.64 & & & 6.869 & 14 & 246 & 275.35 & 131 \\
\hline
\end{tabular}

146 
273.15

273.15

273.15

\section{Table 3}

170 Estimated reservoir temperatures of the hot springs in the Simao Basin.

\begin{tabular}{|c|c|c|c|c|c|c|c|c|c|}
\hline \multirow{2}{*}{$\begin{array}{c}\text { Sample } \\
\text { No. }\end{array}$} & \multicolumn{4}{|c|}{ Reservoir temperature $\left({ }^{\circ} \mathrm{C}\right)$} & \multirow{2}{*}{$\begin{array}{c}\text { Sample } \\
\text { No. }\end{array}$} & \multicolumn{4}{|c|}{ Reservoir temperature $\left({ }^{\circ} \mathrm{C}\right)$} \\
\hline & Quartz & $\mathrm{Na}-\mathrm{K}$ & $\mathrm{K}-\mathrm{Mg}$ & $\mathrm{Na}-\mathrm{K}-\mathrm{Ca}$ & & Quartz & $\mathrm{Na}-\mathrm{K}$ & $\mathrm{K}-\mathrm{Mg}$ & $\mathrm{Na}-\mathrm{K}-\mathrm{Ca}$ \\
\hline $1 \mathrm{a}$ & 91 & 172 & 83 & 176 & $11 \mathrm{a}$ & 87 & 139 & 52 & 74 \\
\hline $1 b$ & 92 & 129 & 68 & 90 & $11 b$ & 85 & 120 & 42 & 48 \\
\hline $1 \mathrm{c}$ & 78 & 143 & 70 & 89 & $11 \mathrm{c}$ & 62 & 119 & 42 & 49 \\
\hline $1 d$ & 91 & 127 & 66 & 128 & $11 \mathrm{~d}$ & 55 & 120 & 41 & 51 \\
\hline $2 \mathrm{a}$ & 116 & 151 & 83 & 144 & $12 \mathrm{a}$ & 102 & 108 & 73 & 121 \\
\hline $2 b$ & 117 & 100 & 68 & 75 & $12 \mathrm{aa}$ & 85 & 149 & 92 & 151 \\
\hline $2 c$ & 111 & 115 & 74 & 83 & $12 \mathrm{~b}$ & 111 & 92 & 70 & 90 \\
\hline $2 d$ & 116 & 101 & 68 & 83 & $12 \mathrm{c}$ & 100 & 86 & 69 & 90 \\
\hline $3 b$ & 85 & 84 & 77 & 105 & $12 \mathrm{~d}$ & 100 & 87 & 70 & 91 \\
\hline $3 c$ & 83 & 78 & 79 & 101 & $13 \mathrm{a}$ & 91 & 115 & 42 & 56 \\
\hline
\end{tabular}

$$
\theta_{\mathrm{KMg}}\left({ }^{\circ} \mathrm{C}\right)=\frac{4410}{14.0-\log \left[\frac{\mathrm{K}^{2}}{\mathrm{Mg}}\right]}-
$$

$$
\theta_{\mathrm{NaK}}\left({ }^{\circ} \mathrm{C}\right)=\frac{1217}{1.483-\log \left[\frac{\mathrm{Na}}{\mathrm{K}}\right]}-
$$

$$
\theta_{\mathrm{NaKCa}}\left({ }^{\circ} \mathrm{C}\right)=\frac{1647}{\log \left[\frac{\mathrm{Na}}{\mathrm{K}}\right]+\beta[\log (\sqrt{\mathrm{Ca}} / \mathrm{Na})+2.06]+2.47}-
$$

\section{Reservoir temperature and variations}




\begin{tabular}{|c|c|c|c|c|c|c|c|c|c|}
\hline $3 \mathrm{~d}$ & 81 & 78 & 79 & 102 & $13 \mathrm{aa}$ & 74 & 114 & 40 & 55 \\
\hline $4 \mathrm{a}$ & 97 & 101 & 81 & 118 & $13 b$ & 85 & 86 & 33 & 33 \\
\hline $4 b$ & 100 & 71 & 66 & 90 & $13 \mathrm{c}$ & 77 & 86 & 32 & 36 \\
\hline $4 c$ & 67 & 63 & 67 & 86 & $13 \mathrm{~d}$ & 82 & 84 & 31 & 38 \\
\hline $4 d$ & 95 & 63 & 67 & 87 & $14 \mathrm{a}$ & 117 & 202 & 106 & 197 \\
\hline $5 \mathrm{a}$ & 91 & 97 & 91 & 118 & $14 \mathrm{c}$ & 111 & 180 & 102 & 164 \\
\hline $5 b$ & 88 & 71 & 75 & 94 & $14 \mathrm{~d}$ & 103 & 170 & 97 & 162 \\
\hline $5 c$ & 86 & 67 & 83 & 94 & $15 c$ & 138 & 166 & 97 & 153 \\
\hline $5 \mathrm{~d}$ & 92 & 67 & 82 & 94 & $15 \mathrm{~d}$ & 124 & 166 & 96 & 153 \\
\hline $6 a$ & 83 & 157 & 38 & 28 & $16 \mathrm{a}$ & 134 & 224 & 96 & 200 \\
\hline $6 \mathrm{~b}$ & 82 & 142 & 33 & 17 & $16 c$ & 128 & 201 & 89 & 170 \\
\hline $6 c$ & 63 & 142 & 33 & 16 & $16 \mathrm{~d}$ & 124 & 204 & 89 & 171 \\
\hline $6 \mathrm{~d}$ & 77 & 136 & 32 & 21 & $17 \mathrm{c}$ & 134 & 186 & 98 & 163 \\
\hline $7 \mathrm{a}$ & 107 & 207 & 79 & 185 & $17 \mathrm{~d}$ & 125 & 187 & 99 & 165 \\
\hline $7 \mathrm{aa}$ & 99 & 194 & 79 & 164 & $18 \mathrm{a}$ & 146 & 205 & 75 & 72 \\
\hline $7 \mathrm{~b}$ & 111 & 193 & 73 & 91 & $18 \mathrm{~b}$ & 138 & 190 & 68 & 51 \\
\hline $7 \mathrm{c}$ & 91 & 248 & 83 & 87 & $18 \mathrm{c}$ & 120 & 190 & 67 & 51 \\
\hline $7 \mathrm{~d}$ & 104 & 195 & 72 & 163 & $18 \mathrm{~d}$ & 128 & 189 & 67 & 50 \\
\hline $8 \mathrm{a}$ & 69 & 191 & 12 & 109 & $19 b$ & 133 & 223 & 65 & 46 \\
\hline $8 \mathrm{~b}$ & 62 & 258 & 14 & 131 & $19 \mathrm{c}$ & 99 & 215 & 55 & 45 \\
\hline $8 \mathrm{c}$ & 51 & 260 & 14 & 131 & $19 \mathrm{~d}$ & 130 & 214 & 55 & 44 \\
\hline $8 \mathrm{~d}$ & 79 & 255 & 13 & 130 & $20 \mathrm{~b}$ & 162 & 222 & 88 & 75 \\
\hline $9 \mathrm{a}$ & 57 & 94 & - & 59 & $20 \mathrm{c}$ & 149 & 223 & 87 & 75 \\
\hline $9 b$ & 48 & 195 & 15 & 107 & $20 \mathrm{~d}$ & 156 & 223 & 87 & 75 \\
\hline $9 \mathrm{c}$ & 32 & 198 & 15 & 107 & $21 \mathrm{a}$ & 177 & 216 & 111 & 196 \\
\hline $9 \mathrm{~d}$ & 67 & 200 & 16 & 111 & $21 \mathrm{~b}$ & 164 & 221 & 91 & 182 \\
\hline $10 \mathrm{a}$ & 102 & 168 & 70 & 149 & $21 \mathrm{c}$ & 154 & 221 & 92 & 183 \\
\hline $10 \mathrm{~b}$ & 100 & 139 & 66 & 67 & $21 \mathrm{~d}$ & 153 & 223 & 91 & 180 \\
\hline $10 \mathrm{c}$ & 74 & 139 & 58 & 69 & & & & & \\
\hline $10 \mathrm{~d}$ & 88 & 140 & 59 & 74 & & & & & \\
\hline
\end{tabular}

171

173 those by cation geothermometers are $63-260^{\circ} \mathrm{C}(\mathrm{Na}-\mathrm{K}), 12-91^{\circ} \mathrm{C}(\mathrm{Na}-\mathrm{K}), 16-200(\mathrm{Na}-\mathrm{K}-\mathrm{Ca})$,

175 equilibrated waters, partially-equilibrated waters, and immature waters, which are used to determine

176 whether a fluid has reached water-rock equilibrium [22]. Only fully-equilibrated waters are suitable for

177 use with cation geothermometers. In Fig. 5, most data points for the water samples from the hot springs

178 plot in the area of immature water. Samples No. 2, 3, 4, 5, and 12 are located in the partially-equilibrated

179 area of the triangular diagram. This suggests that the main cation concentrations in the geothermal water 180 and the wall rock have not reached equilibrium due to the addition of a significant quantity of cold water 


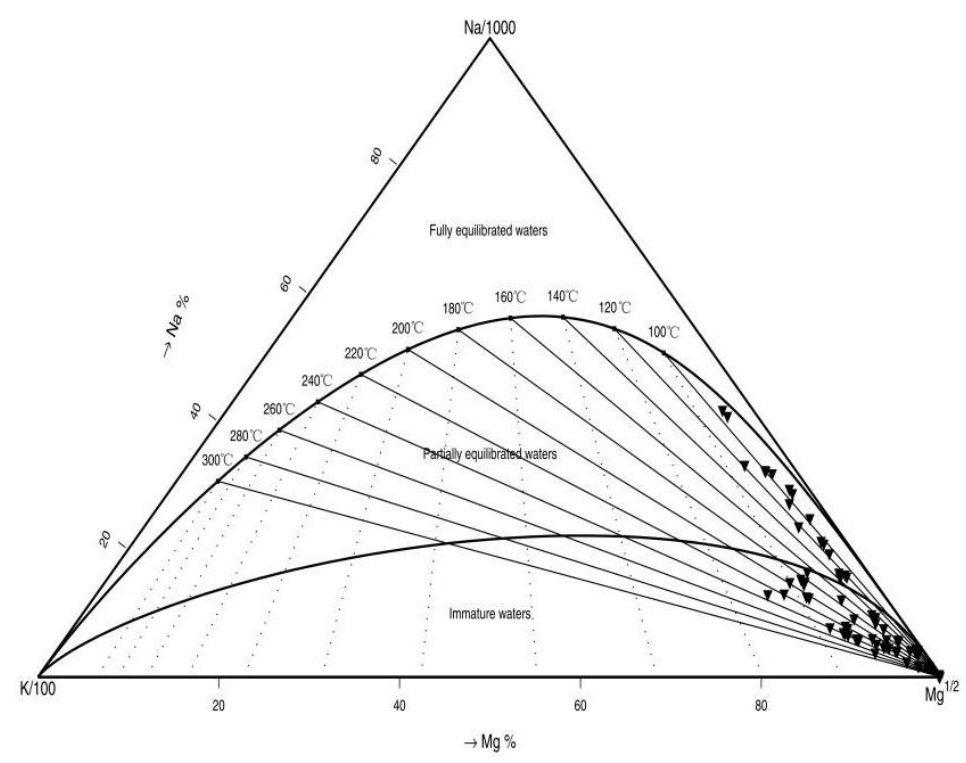

Fig. 5. The Na-K-Mg trilinear equilibrium diagram of the hydrothermal water from the Simao basin.

The calculated saturation index (SI) of various minerals in water as a function of temperature in the Simao Basin suggests that the quartz geothermometer provides more reasonable estimations of the reservoir temperature than cation geothermometers in this area [11]. As mentioned previously, the studied hot spring waters are mixtures of thermal groundwater with shallow cold waters, which lead to a highly dilute silica concentration. Therefore, the temperatures calculated with $\mathrm{SiO}_{2}$ geothermometers were lower than the most reliable reservoir temperatures. The silica-enthalpy mixing model equation and silica-enthalpy diagram can be used to estimate the proportion of the thermal groundwater and cold waters, as well as the original temperature of the thermal groundwater component, which can be used as the reservoir temperatures [24]. The silica-enthalpy equation was used to calculate the proportion of the cold water during mixing based on the dissolved silica contents and enthalpy values of cold and mixed hot waters [25].

$$
\begin{aligned}
& \mathrm{H}_{\mathrm{c}} \cdot \mathrm{X}_{1}+\mathrm{H}_{\mathrm{h}}\left(1-\mathrm{X}_{1}\right)=\mathrm{H}_{\mathrm{s}} \\
& \mathrm{Si}_{\mathrm{c}} \cdot \mathrm{X}_{2}+\mathrm{Si}_{\mathrm{h}}\left(1-\mathrm{X}_{2}\right)=\mathrm{Si}_{\mathrm{s}}
\end{aligned}
$$

where $H_{\mathrm{c}}$ is the enthalpy of near-surface cold water, $H_{\mathrm{h}}$ is the initial enthalpy of deep hot water, $H_{\mathrm{s}}$ is the enthalpy of mixed hot water, $S i_{\mathrm{c}}$ is the silica content of near-surface cold water, $S i_{\mathrm{h}}$ is the initial silica content of deep hot water, and $S i_{\mathrm{S}}$ is the silica content of mixed hot water. $X_{1}$ and $X_{2}$ are the 

and decreased to a relatively stable level after the earthquake $\left(220-260^{\circ} \mathrm{C}\right)$.

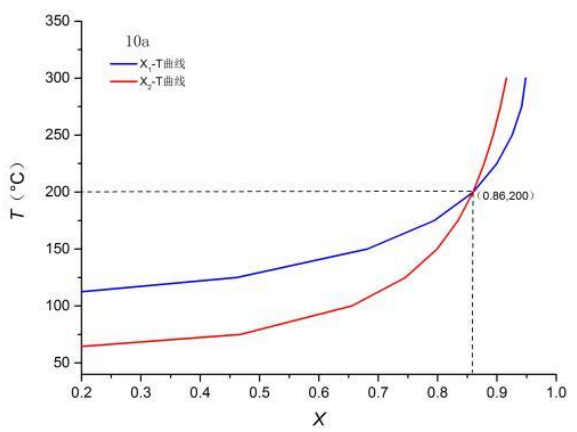
of the deep thermal groundwater (Fig. 7, taking the example of samples 10a-d).

calculated cold water mixing ratios of the enthalpy and the $\mathrm{SiO}_{2}$ concentration, respectively. The solution of equations (5) and (6) was calculated using a diagrammatic method (Fig. 6, taking the example of samples 10a-d) because $X_{1}, X_{2}$, and $H_{\mathrm{s}}$ are unknown. The relationship between $X$ and temperature was plotted to obtain two curves whose intersection gave the estimated proportion of cold water and the original temperature of the thermal groundwater component. The silica-enthalpy diagram was used to estimate the temperature of the deep thermal groundwater with a plot of silica concentration vs. the enthalpy of water [24]. A line through the characteristic points for the cold (point A) and geothermal (point B) waters, the intersection (point C) of the line, and the quartz solubility curve shows the enthalpy

Except for data points $4 \mathrm{c}, 5 \mathrm{c}, 7 \mathrm{c}, 10 \mathrm{c}$, and $12 \mathrm{c}$ at lower temperatures, the reservoir temperatures of the mixing model of the silica-enthalpy equation and diagram ranged between $121{ }^{\circ} \mathrm{C}$ and $289{ }^{\circ} \mathrm{C}$ (Table 4). These values are significantly higher than those of the reservoir temperatures determined by the $\mathrm{SiO}_{2}$ geothermometer in Table 2. The proportions of cold water mixing ranged from $69 \%$ to $98 \%$. There is no intersection point at the plots of samples 1c-d, 6c, 8c, 9a-d, 11c, 14a-d, 16a-d, 18a-d, and 19a-d, indicating that heat was lost before the hot and cold waters mixed. The reservoir temperatures in sampling points $4 \mathrm{c}, 5 \mathrm{c}, 7 \mathrm{c}, 10 \mathrm{c}$, and $12 \mathrm{c}$, which are located approximately $50-150 \mathrm{~km}$ southwest of the epicenters of the M5.9 Mojiang earthquake, showed large decreases after the earthquake (Fig. 8). Especially, the reservoir temperatures of sample $10 \mathrm{c}$ decreased by $\sim 100{ }^{\circ} \mathrm{C}$ after the M5.9 Mojiang earthquake but recovered in $2020\left(200-223^{\circ} \mathrm{C}\right)$. The variations in reservoir temperature are similar to those of the $\mathrm{M}$ 8.0 Wenchuan earthquake [9]. The reservoir temperature before the Wenchuan earthquake was $\sim 340{ }^{\circ} \mathrm{C}$

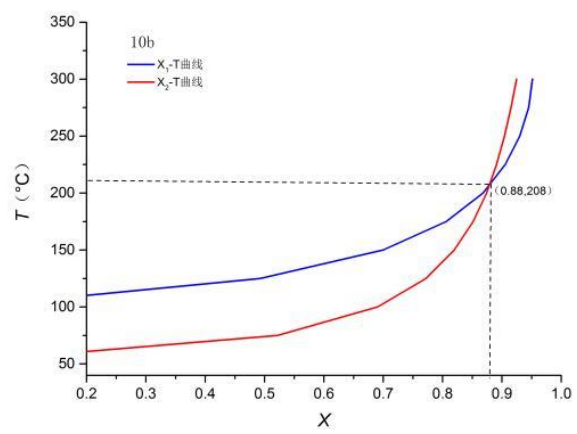



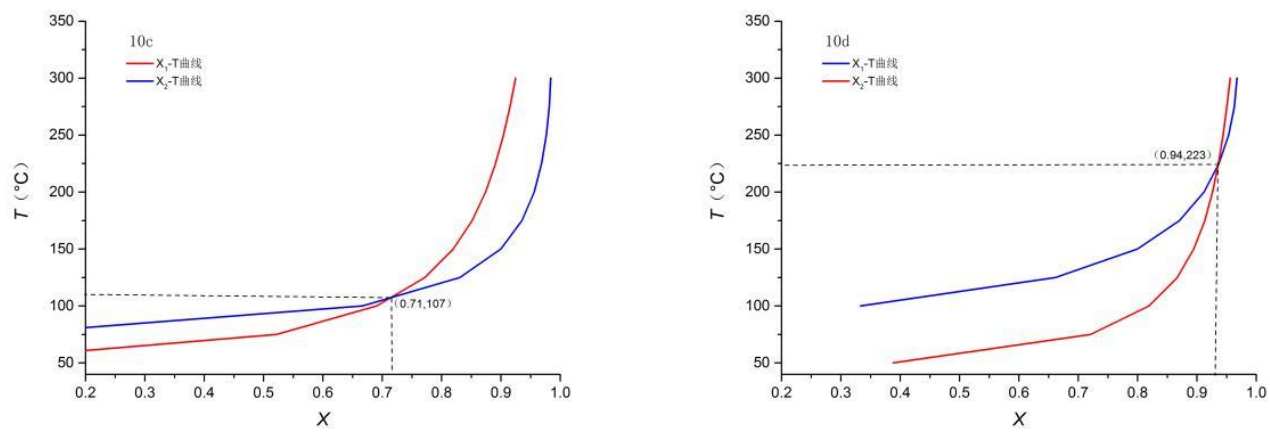

226 Fig. 6. Diagram of temperature relative to the proportion of cold water in hot spring sample $10(X$ is the proportion of the cold water).
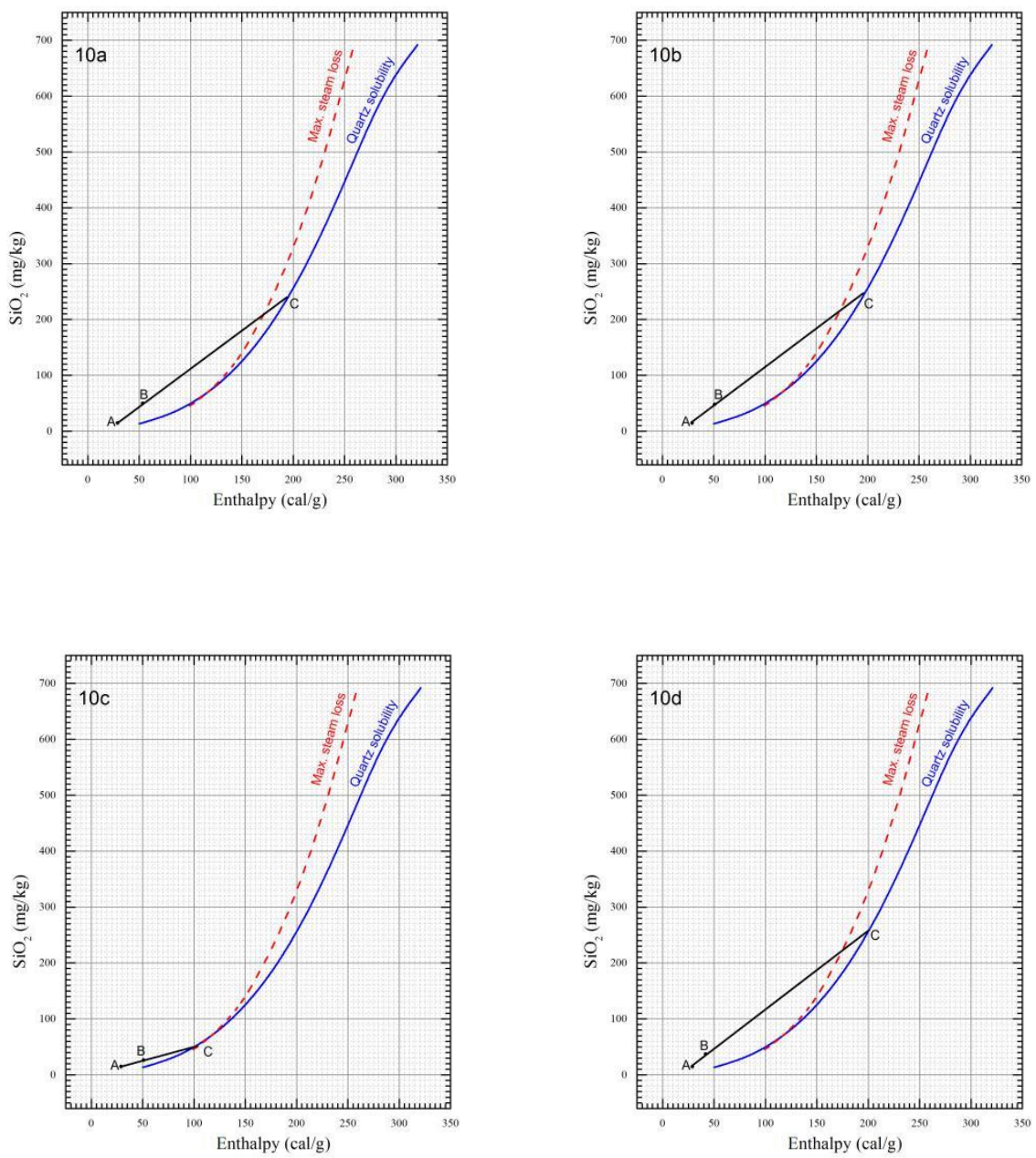


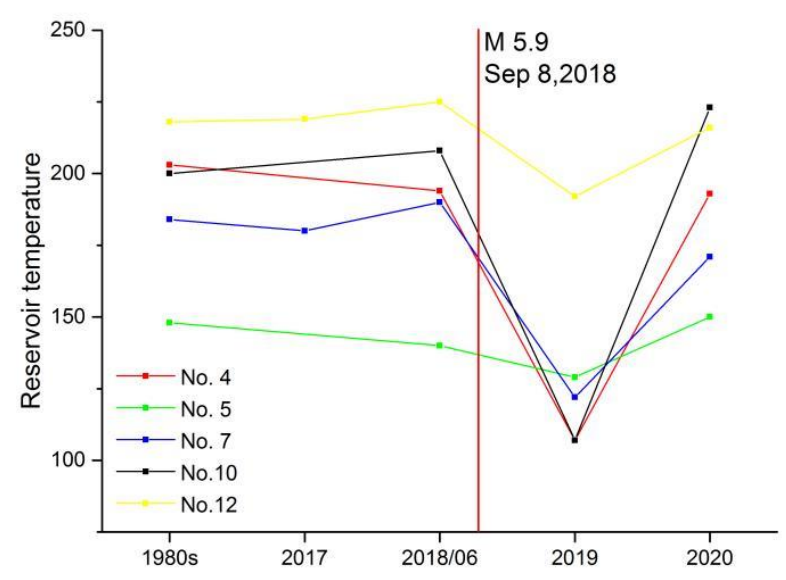

Fig. 8. Reservoir temperature variations in some springs from the 1980s to 2020.

\section{Table 4}

235 Reservoir temperatures based on the silica-enthalpy mixing model and cold water mixing proportions of the hot springs in the Simao Basin.

\begin{tabular}{|c|c|c|c|c|c|c|c|}
\hline \multirow{2}{*}{ Sample } & \multicolumn{2}{|c|}{ Reservoir temperature $\left({ }^{\circ} \mathrm{C}\right)$} & \multirow{2}{*}{$\begin{array}{c}\text { Cold } \\
\text { water } \\
\text { mixing } \\
\text { proportion } \\
(\%)\end{array}$} & \multirow{2}{*}{ Sample } & \multicolumn{2}{|c|}{ Reservoir temperature $\left({ }^{\circ} \mathrm{C}\right)$} & \multirow{2}{*}{$\begin{array}{c}\text { Cold } \\
\text { water } \\
\text { mixing } \\
\text { proportion } \\
(\%)\end{array}$} \\
\hline & $\begin{array}{l}\text { Silica-enthalpy } \\
\text { graph }\end{array}$ & $\begin{array}{l}\text { Silica-enthalpy } \\
\text { equation }\end{array}$ & & & $\begin{array}{l}\text { Silica-enthalpy } \\
\text { graph }\end{array}$ & $\begin{array}{l}\text { Silica-enthalpy } \\
\text { equation }\end{array}$ & \\
\hline 1a & 226 & 220 & 92.6 & $11 \mathrm{a}$ & 231 & 234 & 94.6 \\
\hline $1 b$ & 192 & 194 & 89 & $11 b$ & 231 & 228 & 94.7 \\
\hline $1 \mathrm{c}$ & - & - & - & $11 \mathrm{c}$ & & & \\
\hline $1 \mathrm{~d}$ & - & - & - & $11 \mathrm{~d}$ & & & \\
\hline $2 \mathrm{a}$ & 280 & 275 & 91.3 & $12 \mathrm{a}$ & 225 & 218 & 88.9 \\
\hline $2 b$ & - & - & - & $12 \mathrm{aa}$ & 225 & 219 & 94 \\
\hline $2 \mathrm{c}$ & 272 & 263 & 91.5 & $12 \mathrm{~b}$ & 227 & 225 & 87 \\
\hline $2 \mathrm{~d}$ & - & - & - & $12 \mathrm{c}$ & $188 \downarrow$ & $192 \downarrow$ & 84.7 \\
\hline $3 b$ & 241 & 258 & 96.2 & $12 \mathrm{~d}$ & 200 & 216 & 89.2 \\
\hline $3 \mathrm{c}$ & - & - & - & $13 \mathrm{a}$ & 256 & 247 & 94.7 \\
\hline $3 \mathrm{~d}$ & - & - & - & 13aа & 146 & 154 & 90.6 \\
\hline $4 \mathrm{a}$ & 214 & 203 & 88.5 & $13 b$ & - & - & - \\
\hline $4 \mathrm{~b}$ & 194 & 194 & 85.3 & $13 \mathrm{c}$ & 279 & 263 & 97.6 \\
\hline $4 \mathrm{c}$ & $103 \downarrow$ & $107 \downarrow$ & 81.7 & $13 \mathrm{~d}$ & - & - & - \\
\hline $4 \mathrm{~d}$ & 193 & 193 & 87.2 & $14 \mathrm{a}$ & - & - & - \\
\hline $5 \mathrm{a}$ & 147 & 148 & 76.9 & $14 \mathrm{c}$ & - & - & - \\
\hline
\end{tabular}




\begin{tabular}{|c|c|c|c|c|c|c|c|}
\hline $5 b$ & 142 & 140 & 74.7 & $14 \mathrm{~d}$ & - & - & - \\
\hline $5 c$ & $114 \downarrow$ & $129 \downarrow$ & 71.4 & $15 \mathrm{c}$ & 252 & 246 & 80.9 \\
\hline $5 \mathrm{~d}$ & 150 & 150 & 77.2 & $15 \mathrm{~d}$ & 208 & 206 & 76 \\
\hline $6 a$ & 143 & 151 & 84 & $16 a$ & - & - & - \\
\hline $6 b$ & 140 & 149 & 83.8 & $16 \mathrm{c}$ & - & - & - \\
\hline $6 c$ & - & - & - & $16 \mathrm{~d}$ & - & - & - \\
\hline $6 \mathrm{~d}$ & 121 & 126 & 80.3 & $17 \mathrm{c}$ & 268 & 267 & 85.6 \\
\hline $7 \mathrm{a}$ & 185 & 184 & 78.5 & $17 \mathrm{~d}$ & 239 & 233 & 82.7 \\
\hline 7aа & 154 & 150 & 69.2 & $18 \mathrm{a}$ & - & - & - \\
\hline $7 \mathrm{~b}$ & 187 & 190 & 78.2 & $18 \mathrm{~b}$ & - & - & - \\
\hline $7 \mathrm{c}$ & $122 \downarrow$ & $122 \downarrow$ & 59.3 & $18 \mathrm{c}$ & - & - & - \\
\hline $7 \mathrm{~d}$ & 165 & 171 & 76.2 & $18 \mathrm{~d}$ & - & - & - \\
\hline $8 a$ & 225 & 256 & 98.4 & $19 b$ & - & - & - \\
\hline $8 b$ & 217 & 183 & 97.9 & $19 \mathrm{c}$ & - & - & - \\
\hline $8 c$ & - & - & - & $19 \mathrm{~d}$ & - & - & - \\
\hline $8 \mathrm{~d}$ & - & - & - & $20 \mathrm{~b}$ & - & - & - \\
\hline $9 a$ & - & - & - & $20 \mathrm{c}$ & 289 & 271 & 81.2 \\
\hline $9 b$ & - & - & - & $20 \mathrm{~d}$ & - & - & - \\
\hline $9 \mathrm{c}$ & - & - & - & $21 \mathrm{a}$ & - & - & - \\
\hline $9 \mathrm{~d}$ & - & - & - & $21 \mathrm{~b}$ & 278 & 263 & 73.5 \\
\hline $10 \mathrm{a}$ & 190 & 200 & 85.9 & $21 \mathrm{c}$ & 250 & 235 & 69.4 \\
\hline $10 \mathrm{~b}$ & 197 & 208 & 87.9 & $21 \mathrm{~d}$ & 246 & 237 & 71.1 \\
\hline $10 \mathrm{c}$ & $105 \downarrow$ & $107 \downarrow$ & 71.3 & & & & \\
\hline $10 \mathrm{~d}$ & 200 & 223 & 93.5 & & & & \\
\hline
\end{tabular}

\section{Analysis of hydrogeochemical characteristics}

The chemical characteristics of geothermal water in the Simao Basin show that the main ions in the hot spring water mainly come from the dissolution of minerals during the circulation of surface water. The stratum lithology characteristics in the area control the water chemistry characteristics. Previous data provide a clear understanding of the origin and evolution of major ions in thermal groundwater. $\mathrm{Na}$ in

243 the hot spring waters was derived from the dissolution of Na-feldspar. $\mathrm{Ca}^{2+}\left(\mathrm{Mg}^{2+}\right)$ and $\mathrm{HCO}_{3}{ }^{-}$in the hot spring waters are mainly derived from the dissolution of carbonate minerals, gypsum, and anhydrite in

245 the Triassic rocks. The saline spring flows in the red beds and dissolves large amounts of halite with high $\mathrm{Cl}^{-}$and $\mathrm{Na}^{+}$contents.

The existence of LVZs has been revealed by seismic imaging beneath the Simao Basin within the middle-lower crust at a depth of $15-30 \mathrm{~km}$, and is considered to be possible ductile crustal flow [26-28]. Ductile crustal flow may be the dominant heat source of the hot springs in the Simao Basin. The reservoir temperatures $\left(121-289^{\circ} \mathrm{C}\right)$ were estimated in the Simao Basin. The hot springs with higher reservoir 
temperatures $\left(>250^{\circ} \mathrm{C}\right)$ were mainly located at the margin of the basin (e.g., sample sites $2,13,15,17$, 20, and 21), where the Lancangjiang and Ailaoshan deep faults were well-developed. Fracture is the main channel of hot water circulation, and the deep faults help provide deep heat for the spring waters [29]. Furthermore, in the study area, the fissures were well-developed in the carbonate rocks, while the metamorphic strata exposed in the margin of the basin commonly have a low permeability. As a result, springs emerged in the Ailaoshan metamorphic rocks with higher reservoir temperatures.

\section{Assumptions of earthquake-related hydrogeochemical variation}

Two assumptions have often been used to explain earthquake-related hydrogeochemical changes [10]: (i) hydrogeochemical shifts record accelerated water-rock interactions resulting from an increased reactive surface area caused by fracture of the sampled aquifer; or (ii) hydrogeochemical shifts record the mixing of (or switching between) chemically distinct aquifers. This could occur in response to the fracture of a hydrological barrier between aquifers or because of a change in the relative pressures of connected aquifers.

When an earthquake occurs, the pore pressure in rocks is enhanced as a seismic wave propagates through, causing extensive fracture of the wall rock [30]. This increased the rock surface area in contact with groundwater, which resulted in more extensive water-rock interactions. The saturation index (SI) is commonly used in hydrogeochemistry to indicate the chemical states of mineral phases in groundwater. The saturation state of hot springs in the Simao Basin was calculated based on the chemical compositions and physicochemical parameters of the hydrothermal waters via the PHREEQC program package [31]. The SI values of carbonate minerals (calcite and aragonite) exhibited significant changes before and after the Mojiang earthquake (Fig. 9). The SI values of other selected minerals (e.g., gypsum, anhydrite, celestite, and halite) were less than 0 , remaining undersaturated. The calcite and aragonite saturation trends are shown in Figure 9. The SI values of calcite and aragonite in almost all undersaturated sampling points reached saturation before the Mojiang M5.9 earthquake, indicating the enhanced dissolution of carbonate rocks. This could explain the observed increases in the concentrations of $\mathrm{Ca}^{2+}$ and $\mathrm{HCO}_{3}{ }^{-}$ during the Mojiang earthquake on 8 September 2018. The water chemistry type in No.7 and 13 changed from $\mathrm{Na}-\mathrm{HCO}_{3}-\mathrm{Cl}$ to $\mathrm{Na}-\mathrm{Ca}-\mathrm{HCO}{ }_{3}, \mathrm{Na}-\mathrm{Ca}-\mathrm{Cl}-\mathrm{SO}_{4}-\mathrm{HCO}_{3}$ to $\mathrm{Na}-\mathrm{Ca}-\mathrm{HCO}_{3}-\mathrm{Cl}_{-} \mathrm{SO}_{4}$, which also indicates that $\mathrm{Ca}^{2+}$ and $\mathrm{HCO}_{3}{ }^{-}$dissolution was in-progress. After the earthquake, the SI ratios continued to increase to oversaturation, indicating that precipitation occurred in hot springs, and the dissolved ion 


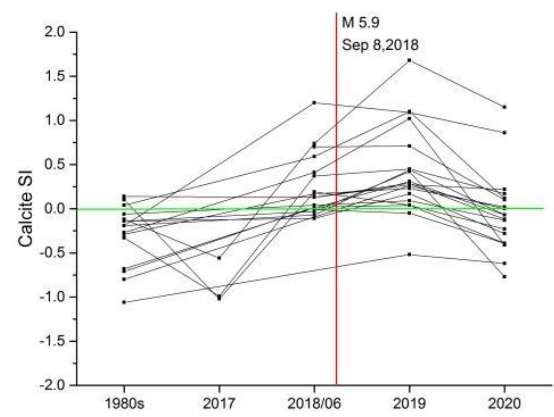

concentration decreased. The SI values decreased and recovered in 2020. This progressive reequilibration by water-rock interactions explained why the $\mathrm{Ca}^{2+}$ and $\mathrm{HCO}_{3}{ }^{-}$contents returned to their pre-seismic levels and why the water chemistry type in No.7 and 13 also recovered.
285

Fig. 9. Variations in the SIs of calcite and aragonite in the hot springs from the 1980s to 2020.

The rupture of hydrological barriers or permeability changes are probable causes of variations in ion concentrations and reservoir temperature. Under the N-S-trending tectonic compression stresses, the local stress concentration may give rise to a new fracture and induce moderately-large earthquakes like the Mojiang M5.9 earthquake [32]. New fractures were created during the Mojiang earthquake, which caused the permeability to greatly increase. The obvious increases in the concentrations of $\mathrm{Ca}^{2+}$ and $\mathrm{HCO}_{3}{ }^{-}$may be attributed to a second fluid rich in $\mathrm{Ca}^{2+}$ and $\mathrm{HCO}_{3}{ }^{-}$from the underlying carbonate strata. The variation may correlate with the contribution of deep fluids. Under the Simao-Pu'er seismic zone, strong low-velocity zones are visible, suggesting the existence of an upwelling asthenosphere in the uppermost mantle. High $\mathrm{He}$ isotope signatures $\left({ }^{3} \mathrm{He} /{ }^{4} \mathrm{He}=5.75-8.36 \mathrm{Ra}\right.$; mantle-derived $\left.\mathrm{He}>70 \%\right)$ have been observed in this region [33]. Increased ${ }^{3} \mathrm{He} /{ }^{4} \mathrm{He}$ ratios in hot springs were observed before $\mathrm{M}>4$ earthquakes in the Simao-Pu'er seismic zone, indicating that much more deep fluid was added to these springs.

Moreover, similar to the explanation of the M 8.0 Wenchuan-related reservoir temperature change [9], variations in reservoir temperature after the Mojiang earthquake may have been caused by the entrance of cold shallow groundwater into the reservoir, which participated in deep circulation; however, the clogging of fractures prevented the entry of shallow cold groundwater, and the reservoir temperature increased. 


\section{Conclusions}

We studied the hydrochemical characteristics of 21 thermal springs from 2018 to 2020 in the Simao Basin. These water samples were roughly divided into three groups: saline spring waters, $\mathrm{HCO}_{3}{ }^{-}$-rich spring waters, and $\mathrm{SO}_{4}{ }^{2-}$-rich spring waters. Stratum lithology characteristics in the area controlled the water chemistry characteristics. $\mathrm{Ca}^{2+}\left(\mathrm{Mg}^{2+}\right)$ and $\mathrm{HCO}_{3}{ }^{-}$in the hot spring waters were mainly derived from the dissolution of carbonate minerals, gypsum, and anhydrite in Triassic rocks. The saline springs flowed in the red beds and dissolved a large amount of halite with high $\mathrm{Cl}^{-}$and $\mathrm{Na}^{+}$contents. The Ailaoshan metamorphic rocks contain pyrite, which may increase the $\mathrm{SO}_{4}{ }^{2-}$ content in the hot spring waters. The reservoir temperatures $\left(121-289^{\circ} \mathrm{C}\right)$ were estimated in the Simao Basin. It is speculated that the ductile crustal flow could be the dominant heat source of the hot springs of Simao Basin. The hot springs with higher reservoir temperatures $\left(>250^{\circ} \mathrm{C}\right)$ were mainly located in the margin of the basin, which may correlate with nearby deep faults that provide deep heat and metamorphic rocks with a low permeability.

During the study period, the 2018 Mojiang M5.9 earthquake occurred in the study area and caused significant hydrochemical changes. The concentrations of $\mathrm{Ca}^{2+}$ and $\mathrm{HCO}_{3}^{-}$in most springs increased obviously before the Mojiang M5.9 earthquake but decreased after the earthquake. The chemistry types of the two spring samples also changed before the earthquake and eventually recovered to their preseismic types. The reservoir temperature decreased after the earthquake. These hydrogeochemical variations might be attributable to accelerated water-rock interactions and/or the mixing of a second fluid due to the earthquake.

\section{Acknowledgments}

This research is financially supported by the project of Science for Earthquake Resilience of China (XH20053Y).

\section{Authors' contributions}

Qilin Li: Conceptualization, Investigation, Writing - original draft, Visualization. Ciping Zhao: Methodology, Data curation. Yun Wang: Investigation, Discussion. Yiying Zhou and Hua Ran: Investigation.

\section{Funding}

This study was supported by the project of Science for Earthquake Resilience of China (XH20053Y). 


\section{Availability of data}

All underlying data of the research study are included in the manuscript in the form of Tables $1-2$.

\section{Competing interest}

The authors declare that they have no known competing financial interests or personal relationships that could have appeared to influence the work reported in this paper.

\section{References}

1. Zhang YS, Gao DL, Shi L, Liu Y, Zhang XY, Li BL (2013) Hydrochemical Characteristics of Salt Spring and Potassium Prospecting Research in Mengla Salt-belts of Simao Basin, Yunnan. Journal of Salt Lake Research 21:10-17

2. Bo Y, Liu C, Zhao Y, Wang L (2015) Chemical and isotopic characteristics and origin of spring waters in the Lanping Simao Basin, Yunnan, Southwestern China. Chemie der Erde 75:287-300

3. Zhang YQ, Zhou X, Liu HS, Tan MR, Hai K, Yu MX, Huo DX (2018) Hydrogeological characteristics of the hot springs and salty springs occurring in the redbeds in the Lanping-Simao Basin of Yunnan. Hydrogeology and Engineering Geology 45:40-48

4. Zhang YQ, Zhou X, Liu HS, Tan MR, Yu MX, Hai K, Huo DX (2019) Hydrogeochemistry, Geothermometry, and Genesis of the Hot Springs in the Simao Basin in Southwestern China. Geofluids 1423:1-23

5. Cheng Y (2017) The characteristics and tectonic implications of electrical structure beneath the Pu' er area (in Chinese). Recent Dev World Seismol 12:1-23

6. Tsunogai U, Wakita H (1995) Precursory chemical changes in ground water: Kobe earthquake, Japan. Science 269:61-63

7. Claesson L, Skelton A, Graham C, Dietl C, Mörth M, Torssander P, Kockum I (2004) Hydrogeochemical changes before and after a major earthquake. Geology 32:641-644

8. Chen Z, Zhou X, Du J, Xie C, Liu L, Li Y, Yi L, Liu H, Cui Y (2015) Hydrochemical characteristics of hot spring waters in the Kangding district related to the Lushan MS $=7.0$ earthquake in Sichuan, China. Natural Hazards \& Earth System Sciences Discussions 2:1149-1156

9. Li B , Shi Z , Wang G , Liu C (2019) Earthquake-related hydrochemical changes in thermal springs in the Xianshuihe Fault zone, Western China. Journal of Hydrology 579:124175

10. Claesson L, Skelton A, Graham C, Mörth C (2007) The timescale and mechanisms of fault sealing and water-rock interaction after the earthquake. Geofluids 7:427-440

11. Zhang L, Liu YW, Ren HW, Ke YL (2019) Application of Hydrochemistry to the verification of groundwater anomalies (in Chinese). Earthquake 39: 29-38

12. Zhang Y, Zhou X, Liu H, Fang B (2020) Geochemistry of rare earth elements in the hot springs in the Simao Basin in southwestern China. Environmental Earth Sciences79:121

13. Yunnan Bureau of Geology and Mineral Resources (1986) Geology of he Saline Deposits in Simao District. Geologsical Publishing House, Yunnan, Beijing

14. Qu YH, Yuan PQ, Suai KY et al. (1998) Potash- forming rules and prospect of lower tertiary in Lanping- Simao Basin. Geological Publishing House, Yunnan, Beijing 15. Yunnan Bureau of Geology and Mineral Resources (1990) Regional Geology of Yunnan Province, Geological Publishing House, Beijing 
16. Zhou X, Jin X, Liang S, Shen Y, Zhang H (2017) Special topics on groundwater sciences (in Chinese), 2nd edn. Geological Publishing House, Beijing

17. Xu Q, Wang J, Wang J, Zhang W (1992) Terrestrial heat flow and its tectonic significance in Yunnan, China (in Chinese). Geotecton Metallog 16:285-299

18. Luo TX (2007) The Maanshan titanomagnetite deposit in Jinping, Yunnan. Yunnan Geology 1: 124128

19. Ellis AJ (1970) Quantitative interpretation of chemical characteristics of hydrothermal systems. Geothermics 2:516 - 528

20. Wang J, Xiong L, Pang Z (1993) Low-medium temperature convective geothermal system (in Chinese). Science Press, Beijing

21. Fournier RO (1981) Application of water geochemistry to geothermal exploration and reservoir engineering. Geothermal Systems: Principles and Case Histories Jonh Wiley and Sons Ltd 109-143

22. Giggenbach WF (1988) Geothermal solute equilibria: derivation of $\mathrm{Na}-\mathrm{K}-\mathrm{Mg}-\mathrm{Ca}$ geoindicators. Geochim Cosmochim Acta 52:2749-2765

23. Verma SP, Santoyo E (1997) New improved equations for $\mathrm{Na} / \mathrm{K}, \mathrm{Na} / \mathrm{Li}$ and $\mathrm{SiO}_{2}$, geothermometers by outlier detection and rejection. J Volcanol Geotherm Res 79:9-23

24. Fournier RO (1977) Chemical geothermometers and mixing models for geothermal systems. Geothermics 5:41-50

25. Fournier RO, Truesdell AH, Calif MP (1974) Geochemical indicators of subsurface temperature-part 2 , estimation of temperature and fraction of hot water mixed with cold water. Jour Research U.S. Geol Survey 2: 263-270

26. Bai D, Unsworth MJ, Meju MA, Ma XB, Teng JW, Kong XR, Sun Y, Sun J, Wang LF, Jiang CS, Zhao CP, Xiao PF, Liu M (2010) Crustal deformation of the eastern Tibetan Plateau revealed by Magnetotelluric imaging. Nature Geoscience 3:358-362

27. Bao X, Sun X, Xu M, Eaton DW, Song X, Wang L, Ding Z, Mi N, Li H, Yu D (2015) Two crustal low-velocity channels beneath SE Tibet revealed by joint inversion of Rayleigh wave dispersion and receiver functions. Earth and Planetary Science Letters 415:16-24.

28. Li MK, Zhang SX, Wang F, Wu TF, Qin WB (2016) Crustal and upper-mantle structure of the southeastern Tibetan Plateau from joint analysis of surface wave dispersion and receiver function. Journal of Asian Earth Sciences 117: 52-63

29. Tang XC, Zhang J, Pang ZH, Hu SB, Tian J, Bao SJ (2017) The eastern Tibetan Plateau geothermal belt, western China: Geology, geophysics, genesis, and hydrothermal system. Tectonophysics 717:433-448

30. Du J, Zhou X, Chen Z, Cui Y, Liu L, Li Y, Zhang W, Gao X, Xu Q, Wang H (2013) Responses of mud volcanoes in the North Tianshan to the 30 June 2012 Xinyuan-Hejing MS 6.6 earthquake. Acta Seismol Sin 35:876-887

31. Parkhurst DL, Appelo CAJ (1999) User's guide to PHREEQC (version2)-A computer program for speciation, reaction-path, one-dimensional transport, and inverse geochemical calculations. USGS Water Resources Investigation Report 99-4259

32. Chang ZF, Mao ZB, Ma BQ, Dai BY (2019) The Amojiang fault zone and Mojiang M5.9 earthquake in 2018 in southern Yunnan Province. Geological Bulletin of China 38:967-976 
419 Yunnan: genetic significance of M6 earthquake clustering in the Simao-Pu'er seismic zone. Progress

420 in Earthquake Sciences 8:137-139

421 


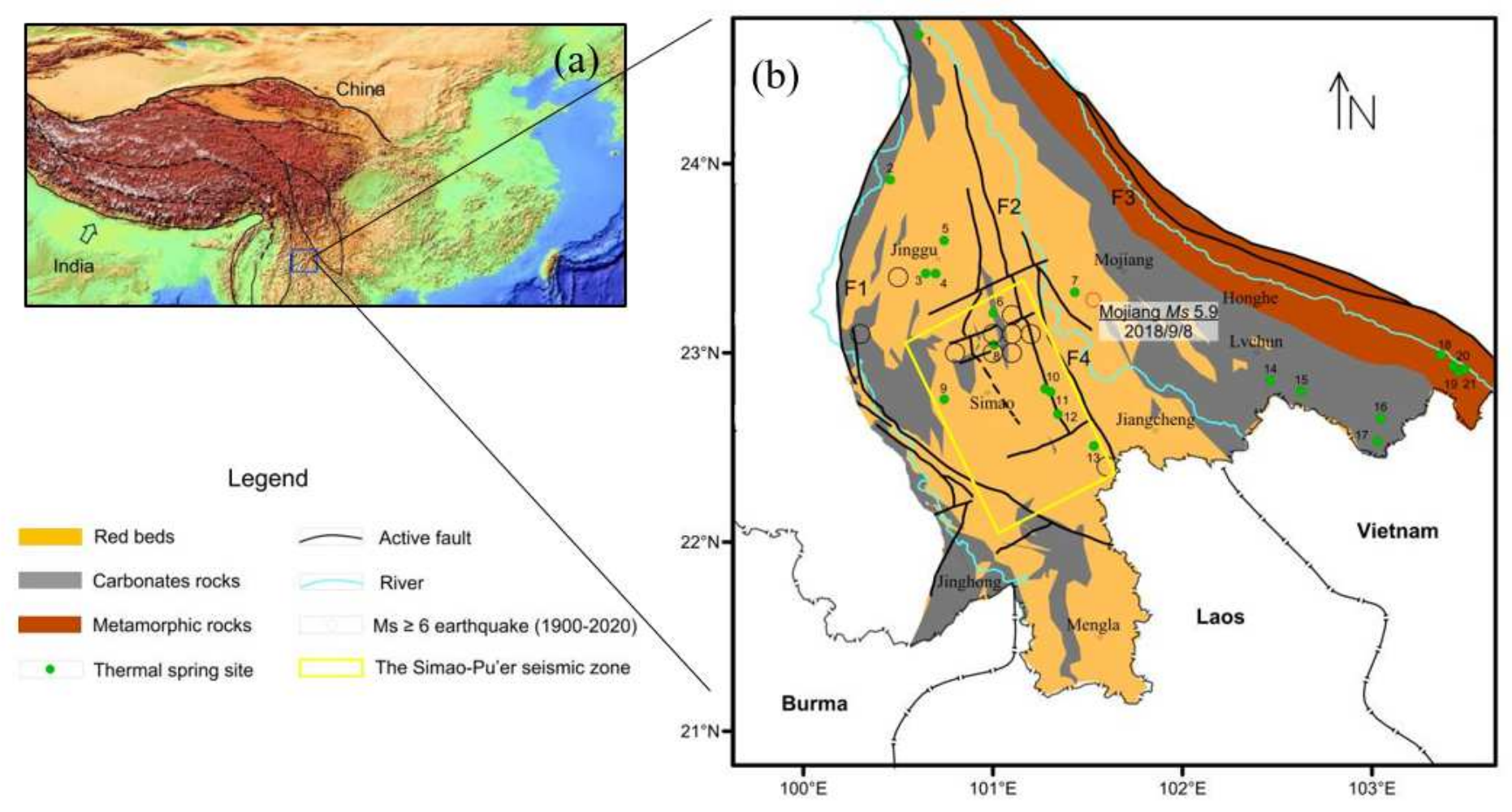

Figure 1

Topographic map of the study area (a) and schematic tectonics and spring sites in the Simao Basin (b; modified from Zhang et al.) [12]. Abbreviations: F1 = Lancangjiang fault, F2 = Wuliangshan fault, F3 = Ailaoshan fault, F4 = Mohei fault. Note: The designations employed and the presentation of the material on this map do not imply the expression of any opinion whatsoever on the part of Research Square concerning the legal status of any country, territory, city or area or of its authorities, or concerning the delimitation of its frontiers or boundaries. This map has been provided by the authors 

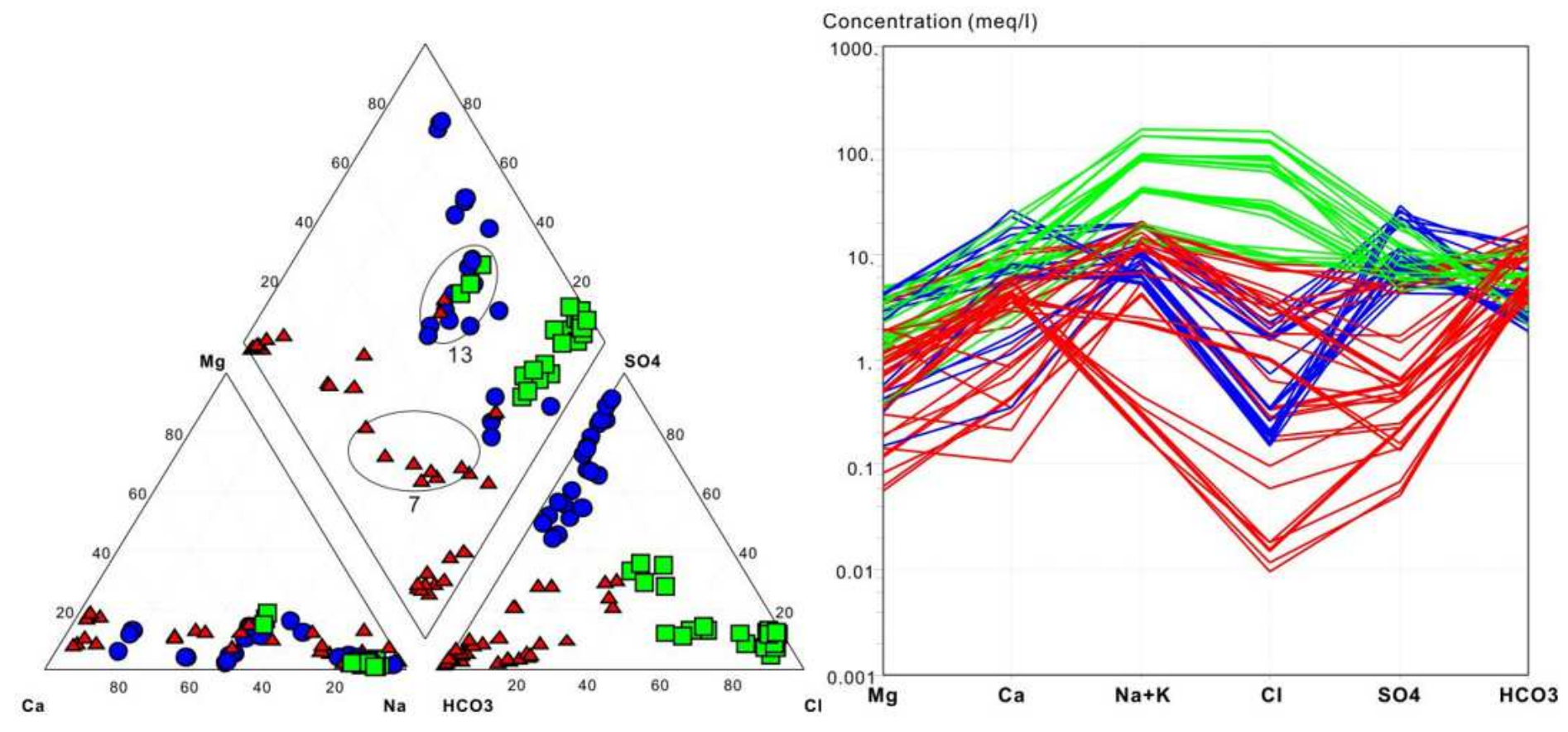

\section{Figure 2}

Piper diagram (a) and Schoeller diagram (b) of the geothermal waters. Process (black circles) indicating the changes before and after the Mojiang earthquake. 

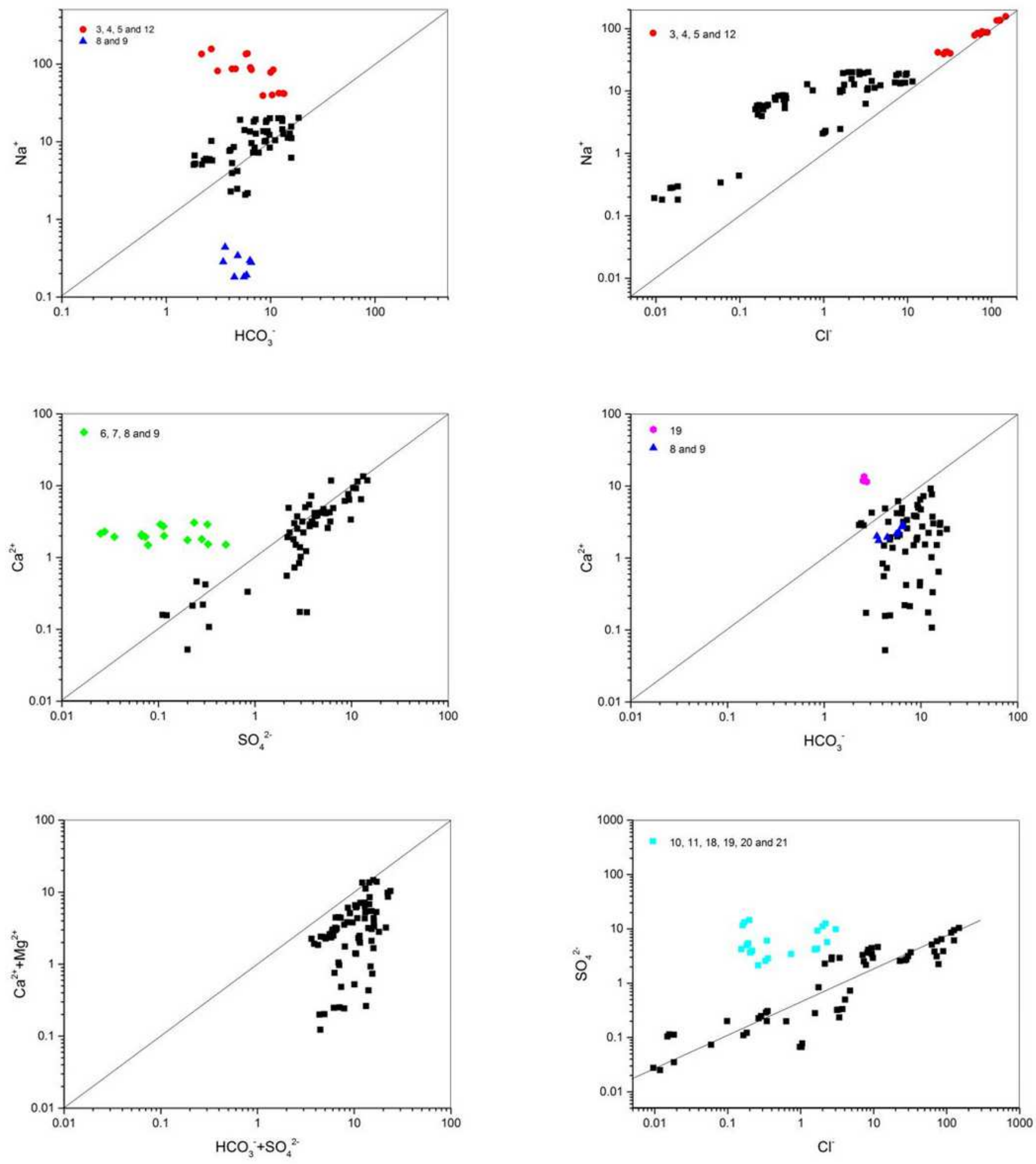

Figure 3

Relationship plots of major ions of the spring water in the Simao Basin. 


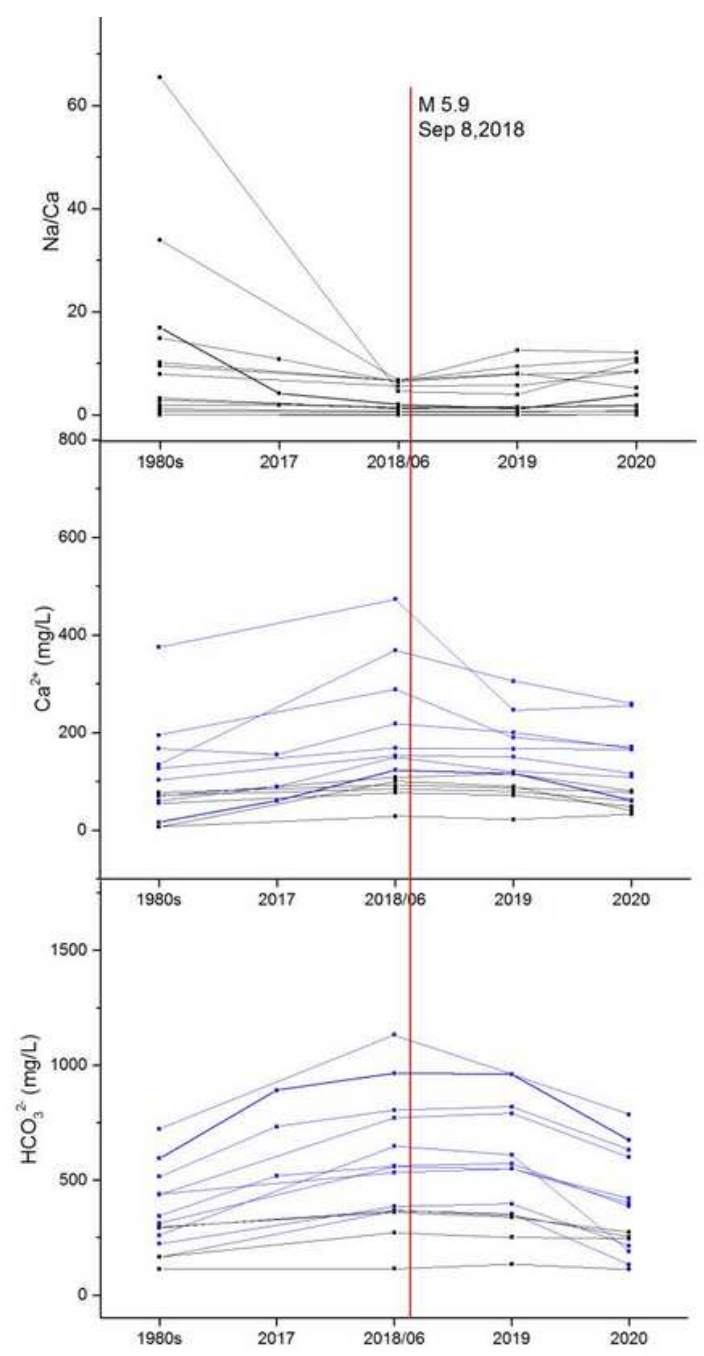

Figure 4

Hydrogeochemical changes of the thermal springs from the 1980 s to 2020 . 


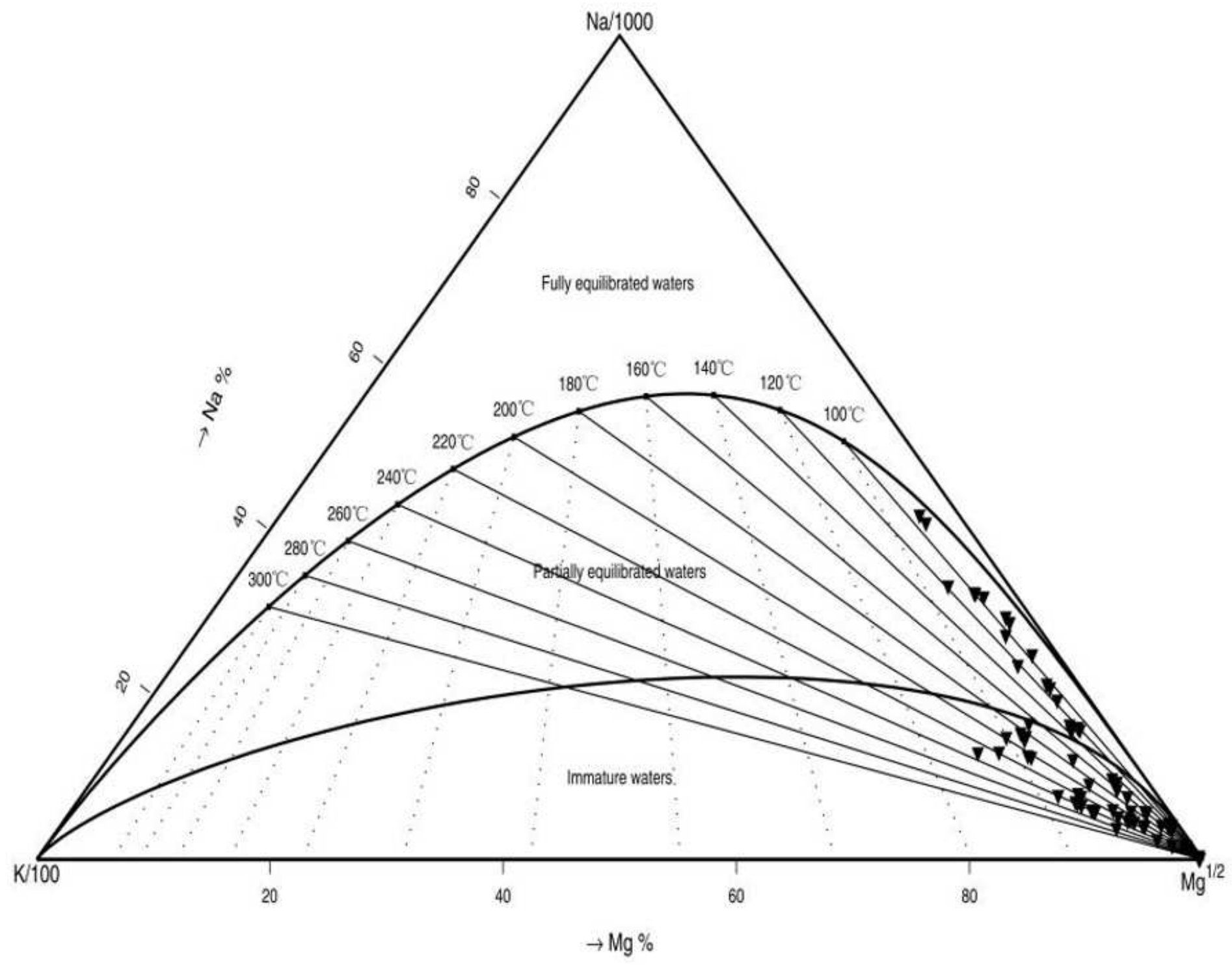

Figure 5

The Na-K-Mg trilinear equilibrium diagram of the hydrothermal water from the Simao basin. 

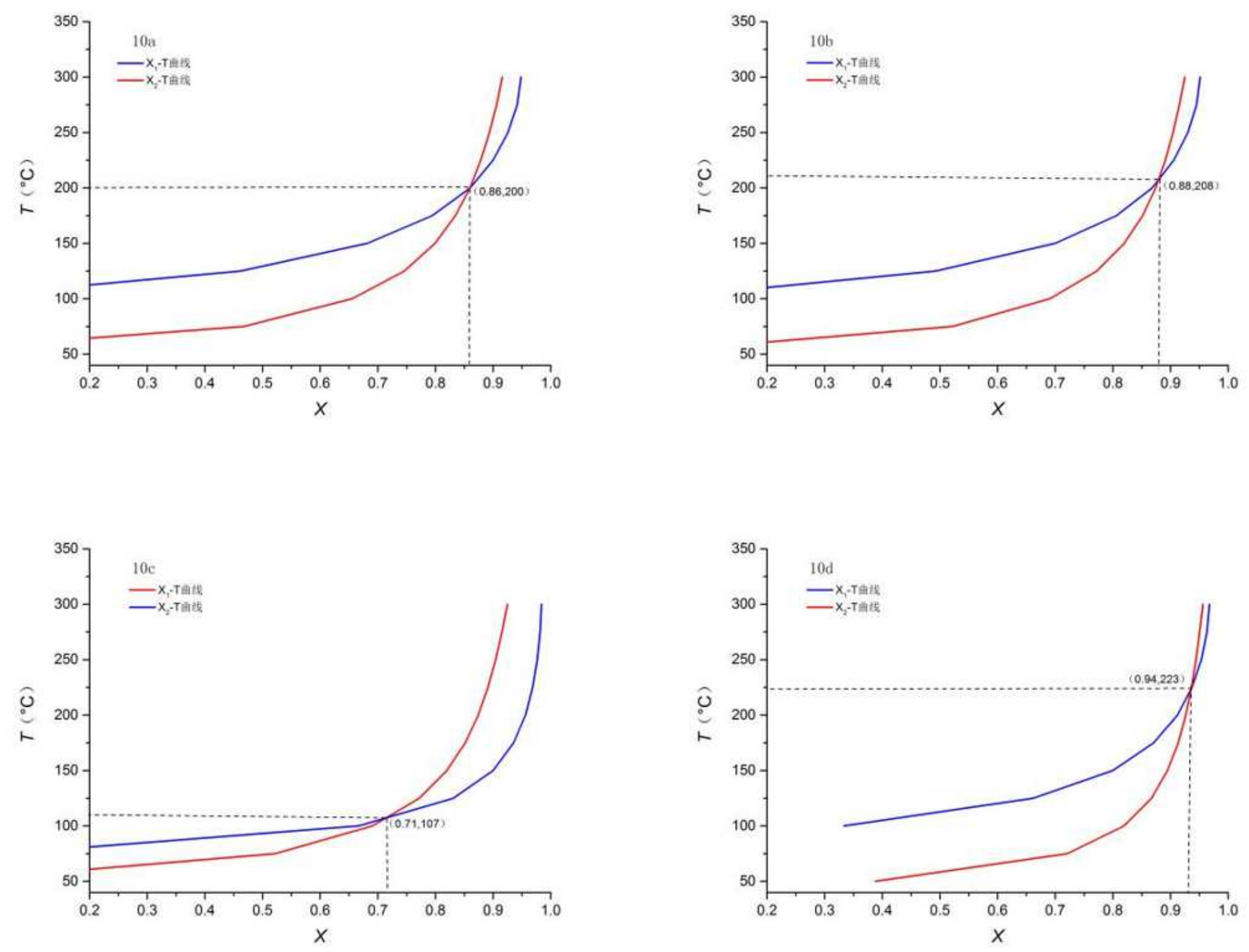

\section{Figure 6}

Diagram of temperature relative to the proportion of cold water in hot spring sample 10 ( $\mathrm{X}$ is the proportion of the cold water). 

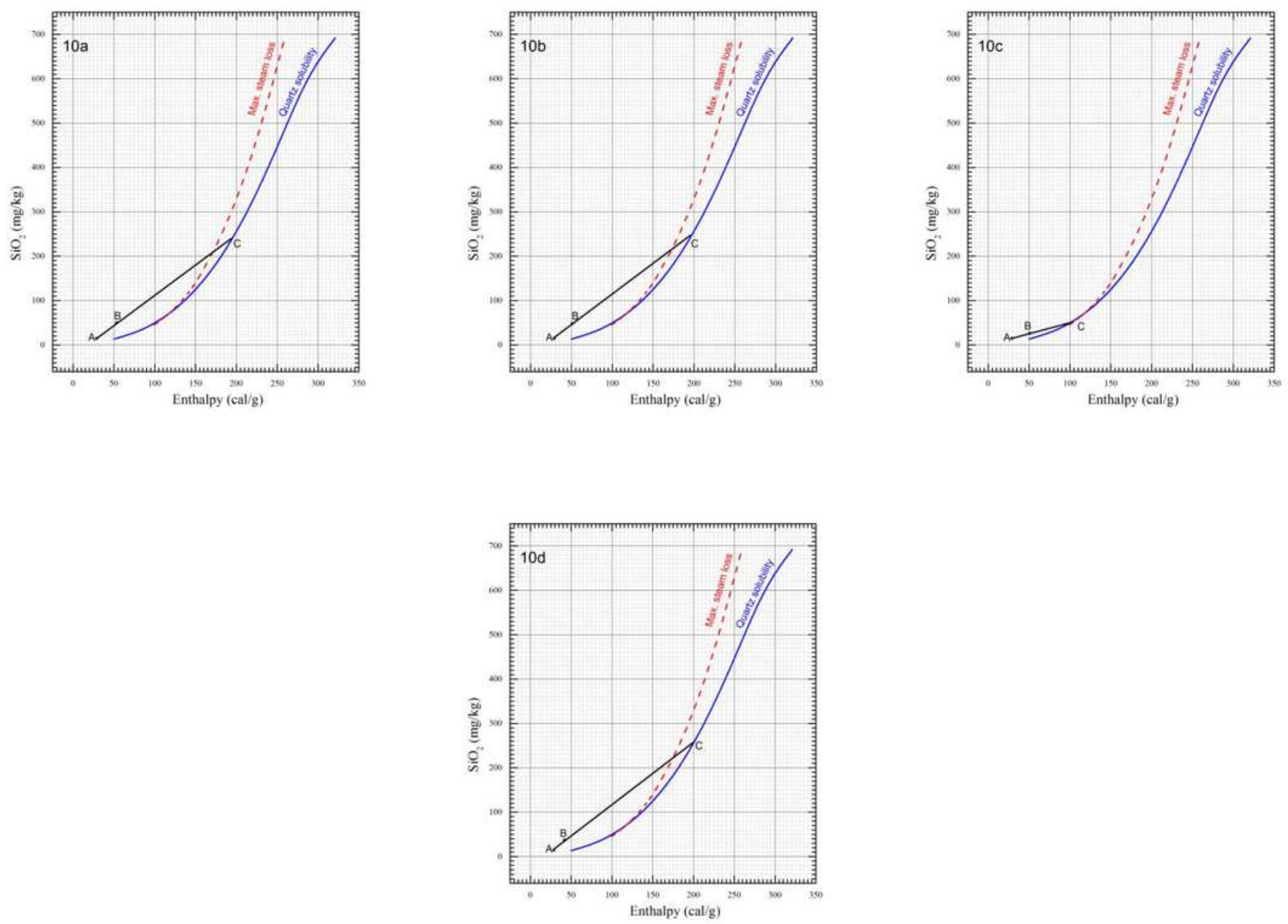

Figure 7

The silica-enthalpy diagram of hot spring sample 10 


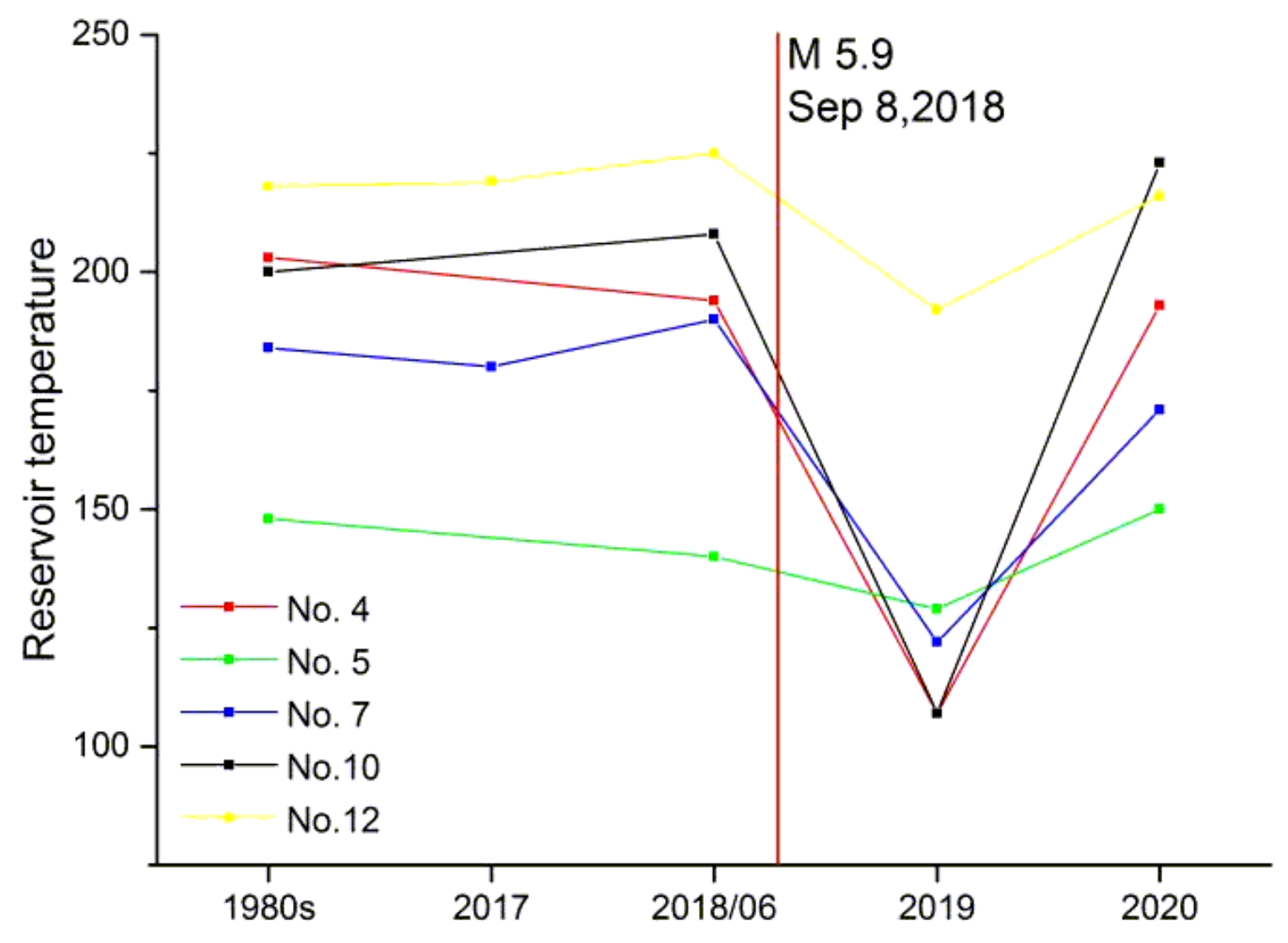

Figure 8

The silica-enthalpy diagram of hot spring sample 10 